\title{
ENTROPY-STABLE SPACE-TIME DG SCHEMES FOR NON-CONSERVATIVE HYPERBOLIC SYSTEMS
}

\author{
Andreas Hiltebrand ${ }^{1}$, Siddhartha Mishra ${ }^{2,3}$ And Carlos Parés ${ }^{4, *}$
}

\begin{abstract}
We propose a space-time discontinuous Galerkin (DG) method to approximate multidimensional non-conservative hyperbolic systems. The scheme is based on a particular choice of interface fluctuations. The key difference with existing space-time DG methods lies in the fact that our scheme is formulated in entropy variables, allowing us to prove entropy stability for the method. Additional numerical stabilization in the form of streamline diffusion and shock-capturing terms are added. The resulting method is entropy stable, arbitrary high-order accurate, fully discrete, and able to handle complex domain geometries discretized with unstructured grids. We illustrate the method with representative numerical examples.
\end{abstract}

Mathematics Subject Classification. 65M60, 65M12, 35L60, 76L05.

Received June 13, 2017. Revised September 28, 2017. Accepted November 9, 2017.

\section{INTRODUCTION}

\subsection{The model}

We consider multi-dimensional systems of hyperbolic partial differential equations (PDEs) of the nonconservative form,

$$
\mathbf{U}_{t}+\sum_{k=1}^{d} A^{k}(\mathbf{U}) \mathbf{U}_{x_{k}}=0 .
$$

Here, the unknown vector $\mathbf{U}=\mathbf{U}\left(x_{1}, \ldots, x_{d}, t\right) \in \mathbb{R}^{N},\left(x_{1}, \ldots, x_{d}\right)$ and $t$ take values in a domain $\Omega \subset \mathbb{R}^{d}$ and $[0, T]$, respectively, and $A^{k}(\mathbf{U}) \in \mathbb{R}^{N \times N}, k=1, \ldots, d$, are smooth matrix-valued functions.

If there exist $d$ flux functions $\mathbf{F}^{1}, \ldots, \mathbf{F}^{d}$ satifsying:

$$
A^{k}(\mathbf{U})=\mathbf{F}_{\mathbf{U}}^{k}, \quad k=1, \ldots, d,
$$

Keywords and phrases: Multidimensional nonconservative hyperbolic systems, space-time discontinuous Galerkin methods, entropy-stability, streamline diffusion, shock-capturing methods, two-layer shallow water system.

1 ANSYS Switzerland GmbH, Zürich 8005, Switzerland.

2 Seminar for Applied Mathematics (SAM), Department of Mathematics, ETH Zürich, HG G 57.2, Zürich 8092, Switzerland.

3 Center of Mathematics for Applications (CMA), University of Oslo, P.O. Box 1053, Blindern, Oslo 0316, Norway.

4 Departamento de Análisis Matemático, Facultad de Ciencias, Universidad de Málaga, 29071 Málaga, Spain.

* Corresponding author: pares@uma.es 
where the subscript $\mathbf{U}$ denotes the Jacobian of a vector function or the gradient of a scalar function, then the system can be written in conservative form

$$
\mathbf{U}_{t}+\sum_{k=1}^{d} \mathbf{F}^{k}(\mathbf{U})_{x_{k}}=0,
$$

resulting in the well-known systems of conservation laws [11]. We are specifically interested in the case in which these flux functions do not exist and one has to consider the general non-conservative form (1.1).

Many problems of interest in physics and engineering are modeled by the non-conservative hyperbolic system (1.1). Prototypical examples include the multi-layer shallow water equations and the governing equations of compressible multi-phase flow.

In light of the above prototypical examples, it is reasonable to assume that the system (1.1) is equipped with an entropy-entropy flux pair $(S, \mathbf{Q})$ where $S: \mathbb{R}^{N} \rightarrow \mathbb{R}$ is a strictly convex function and $\mathbf{Q}=\left(Q^{1}, \ldots, Q^{N}\right)$ is a function from $\mathbb{R}^{N}$ to $\mathbb{R}^{N}$ whose components satisfy the compatibility conditions,

$$
Q_{\mathbf{U}}^{k}(\mathbf{U})=\mathbf{V}^{T} \cdot A^{k}(\mathbf{U})
$$

where

$$
\mathbf{V}=S_{\mathbf{U}}(\mathbf{U})
$$

are the so-called entropy variables.

On account of the strict convexity of $S$, the mapping $\mathbf{U} \rightarrow \mathbf{V}$ is one-to-one. Consequently, one can rewrite the system (1.1) in terms of the entropy variables as

$$
\mathbf{U}(\mathbf{V})_{t}+\sum_{k=1}^{d} \tilde{A}^{k}(\mathbf{V}) \mathbf{V}_{x_{k}}=0
$$

where

$$
\tilde{A}^{k}(\mathbf{V})=A^{k}(\mathbf{U}(\mathbf{V})) \mathbf{U}_{\mathbf{V}}(\mathbf{V})
$$

For notational simplicity, the tilde in (1.5) is dropped subsequently and the equations in the entropy variables are written as

$$
\mathbf{U}(\mathbf{V})_{t}+\sum_{k=1}^{d} A^{k}(\mathbf{V}) \mathbf{V}_{x_{k}}=0
$$

We are interested in approximating entropy solutions of (1.1) or (1.7), i.e. weak solutions that satisfy the entropy inequality

$$
S(\mathbf{V})_{t}+\sum_{k=1}^{d} Q^{k}(\mathbf{V})_{x_{k}} \leq 0
$$

in the sense of distributions. 


\subsection{Mathematical framework}

As is well-known, solutions to the system (1.1) develop discontinuities in the form of shock waves (and contact discontinuities), even when the initial data is smooth. Hence, one needs to interpret the solutions of (1.1) in a weak sense. However, the non-conservative products of the form $A^{k}(\mathbf{U}) \mathbf{U}_{x_{k}}$ cannot be defined in the sense of distributions at the discontinuities of the solution $\mathbf{U}$. A possible solution to this difficulty was proposed in [12], where the authors define these non-conservative products as Borel measures. This theory allows one to define the nonconservative products as bounded measures for functions $\mathbf{U}$ with bounded variation, provided a family of Lipschitz continuous paths $\Phi:[0,1] \times \mathbb{R}^{N} \times \mathbb{R}^{N} \rightarrow \mathbb{R}^{N}$ are prescribed. This family must satisfy certain regularity and compatibility conditions, in particular

$$
\Phi\left(0 ; \mathbf{U}_{-}, \mathbf{U}_{+}\right)=\mathbf{U}_{-}, \quad \Phi\left(1 ; \mathbf{U}_{-}, \mathbf{U}_{+}\right)=\mathbf{U}_{+}, \quad \Phi(s ; \mathbf{U}, \mathbf{U})=\mathbf{U} .
$$

Once the nonconservative product has been defined, one can define the weak solutions of (1.1). In particular, across a discontinuity, a weak solution in the sense of [12], has to satisfy the generalized Rankine-Hugoniot condition

$$
\sigma\left(\mathbf{U}_{+}-\mathbf{U}_{-}\right)=\int_{0}^{1} \sum_{k=1}^{d} A^{k}\left(\Phi\left(s ; \mathbf{U}_{-}, \mathbf{U}_{+}\right)\right) \partial_{s} \Phi\left(s ; \mathbf{U}_{-}, \mathbf{U}_{+}\right) \nu^{k} \mathrm{~d} s
$$

where $\sigma$ is the speed of propagation of the discontinuity, $\mathbf{U}_{-}$and $\mathbf{U}_{+}$are the left and right limits of the solution at the discontinuity, along the normal direction to the shock given by the vector $\vec{\nu}=\left(\nu^{1}, \ldots, \nu^{d}\right)$. We note that (1.9) reduces to the usual Rankine-Hugoniot condition when (1.2) is satisfied and the system (1.1) can be written in the conservation form (1.3).

Unfortunately, the concept of weak solutions as outlined above depends on the chosen family of paths. Different families of paths lead to different jump conditions, and result in different weak solutions. A priori, the choice of paths is arbitrary. Thus, a crucial question is how to choose the correct family of paths in order to recover the physically relevant solution.

In practice, a hyperbolic system such as (1.1) is obtained as the limit of a regularized problem when the high-order terms (corresponding to small-scale effects) are neglected. For instance, it may be the vanishing viscosity $(\epsilon \rightarrow 0)$ limit of the following parabolic system,

$$
\mathbf{U}_{t}+\sum_{k=1}^{d} A^{k}(\mathbf{U}) \mathbf{U}_{x_{k}}=\epsilon \sum_{k, l=1}^{d}\left(R^{l k}(\mathbf{U}) \mathbf{U}_{x_{k}}\right)_{x_{l}}
$$

with an elliptic second-order viscous term. In this case, the correct jump conditions (corresponding to the physically relevant solutions) should be consistent with the viscous profile, i.e. with the traveling wave solutions of the regularized problem (1.10): see [24, 25].

\subsection{Numerical schemes}

A large number of numerical methods have been proposed to approximate the non-conservative hyperbolic system (1.1), see [25] and references therein. A particularly attractive framework is provided by the pathconservative schemes, first proposed in [26]. These schemes are designed to be formally consistent with any particular choice of path for the definition of the non-conservative product and the weak solution for the system (1.1). This framework allows to extend to nonconservative systems well-known families of conservative numerical methods. Moreover, they can be easily extended to high-order of accuracy by using reconstructions operators [6], central schemes [8], discontinuous Galerkin methods [27], ADER methods [13, 14]. A review of the latest developments on path-conservative schemes is provided in [10]. 
However, path-conservative numerical schemes for approximating (1.1) may not converge to the physically relevant weak solution when the mesh is refined. As alluded to in [20] and first demonstrated in [7] (see also [1]), this lack of convergence can be observed even when a path that corresponds to the small-scale limit of the regularized problem (1.10) is chosen (via the viscous profiles of (1.10)) and a path-conservative scheme, consistent with this correct path, is designed. This lack of convergence has been explained in terms of the equivalent (modified) equation, corresponding to the underlying finite difference scheme [3, 7, 23, 25] and references therein. Briefly, the second-order terms of this equivalent equation are determined by the numerical viscosity of the underlying method and do not necessarily match with the physical viscosity in (1.10). Hence, one observes convergence of the resulting scheme towards a different weak solution.

One promising solution to this vexing problem of lack of convergence of path-conservative schemes was provided in [9]. In this article, the authors designed path-conservative finite-difference schemes that were also entropy stable, i.e. they satisfied a discrete version of the entropy inequality (1.8). The construction of these entropy-stable path-conservative schemes was based on the design of path-conservative fluctuations that resulted in a discrete entropy identity and adding suitable amount of numerical viscosity, for instance modeled on the physical viscosity of (1.10). In [9], the authors chose a simple path, corresponding to straight line segments of the form,

$$
\Phi\left(s ; \mathbf{U}_{-}, \mathbf{U}_{+}\right)=\mathbf{U}_{-}+s\left(\mathbf{U}_{+}-\mathbf{U}_{-}\right) .
$$

Numerical experiments showed that this approach was quite successful in providing stability and in approximating the physically relevant weak solution for several prototypical non-conservative systems.

On the other hand, these entropy stable path-conservative schemes of [9] had several limitations namely,

- They were (formally) first-order accurate.

- They were only defined on Cartesian grids in several space dimensions.

- These schemes were semi-discrete. Using standard SSP Runge-Kutta methods to solve the resulting ODEs destroyed the entropy stability properties of the scheme.

\subsection{Aims and scope of the current paper}

Given the above limitations of the entropy-stable path-conservative schemes for approximating the nonconservative hyperbolic system (1.1), our main aim in this paper is to develop a fully discrete, arbitrarily high-order, entropy-stable scheme for approximating multi-dimensional non-conservative hyperbolic systems. We would require that this scheme can also be defined on unstructured grids in-order to be able to handle problems on domains with complex geometry.

To meet these objectives, we propose a shock-capturing streamline-diffusion space-time discontinuous Galerkin (DG) finite element method for approximating (1.1). Such methods were proposed in the context of systems of conservation laws (and convection-diffusion equations) in [2, 21,22] and references therein. Recent results on this discretization framework, in the context of conservation laws, were obtained in [17-19] and references therein.

Space-time DG methods for systems of conservation laws (1.3) consisted of the following design ingredients:

- The numerical method is based on the formulation of the system in entropy variables (1.7).

- An entropy-stable numerical flux is used to deal with the discontinuities appearing at the element boundaries, see for instance $[2,28]$.

- Streamline diffusion and shock-capturing terms are added inside each element to prevent the appearance of unphysical oscillations.

A very similar philosophy is followed in this article. The main difference with the corresponding formulation for systems of conservation laws lies in replacing numerical fluxes across element boundaries, with suitable fluctuations, that correspond to entropy-stable path-conservative formulations of [9]. Hence, these schemes can be considered as generalizations of the entropy-stable path-conservative schemes to arbitrary high-order, 
unstructured grids, and temporal discretizations. We will design suitable fluctuations, streamline diffusion, and shock-capturing operators and prove that the resulting space-time DG method is entropy stable. Numerical examples, illustrating the stability of the method and its ability to converge towards the physically relevant weak solution will also be demonstrated.

As mentioned above, space-time DG methods, based on underlying path-conservative schemes to account for discontinuities of the test functions at spatial element boundaries, have been developed for non-conservative hyperbolic systems, see $[13,14,27]$. There are many similarities between these methods and the one proposed here. However, the key novelty in our method lies in its formulation in terms of entropy variables and the consequent use of entropy stable path-conservative fluctuations. These ingredients are crucial in proving entropy stability for the method. In contrast and to the best of our knowledge, entropy stability cannot be rigorously established for previously developed space-time DG methods, even when entropy-stable path-conservative fluctuations are chosen to take spatial discontinuities into account.

The outline of the paper is as follows: first, the general form of the space-time entropy stable discontinuous Galerkin methods for solving (1.1) is described. Next, the discrete entropy inequality is proved. In Sect. 4 these methods are applied to the 1D and 2D two-layer shallow water equations and the accuracy and the convergence properties of the scheme are investigated. Finally, some conclusions are drawn.

\section{The SPACE-TIME DG SCHEME}

In the following, we specify how the space-time DG formulation of $[17,18]$ for conservation laws can be adapted to non-conservative systems. We start with the definition of the space-time mesh.

\subsection{The mesh}

At the $n$th time level $t^{n}$, we denote the time step as $\Delta t^{n}=t^{n+1}-t^{n}$ and the update time interval as $I^{n}=\left[t^{n}, t^{n+1}\right)$. For simplicity, we assume that the spatial domain $\Omega \subset \mathbb{R}^{d}$ is polyhedral and divide it into a triangulation $\mathcal{T}$, i.e. a set of open convex polyhedra $K \subset \mathbb{R}^{d}$ with plane faces. Furthermore, we assume mesh regularity [21]. For a generic element (cell) $K$, we denote

$$
\begin{aligned}
\Delta x_{K} & =\operatorname{diam}(K) \\
\mathcal{N}(K) & =\left\{K^{\prime} \in \mathcal{T}: K^{\prime} \neq K \wedge \operatorname{meas}_{d-1}\left(\bar{K} \cap \overline{K^{\prime}}\right)>0\right\} .
\end{aligned}
$$

The mesh width of the triangulation is $\Delta x(\mathcal{T})=\max _{K} \Delta x_{K}$. A generic space-time element is the prism:

$$
K \times I^{n} .
$$

We also assume that there exists a constant $C>0$ such that $(1 / C) \Delta x \leq \Delta t^{n} \leq C \Delta x$ for all time levels $n$.

\subsection{Variational formulation}

Following $[2,17,18,28]$, we approximate the equivalent representation of the non-conservative system (1.7) by a DG method. On a given triangulation $\mathcal{T}$ with mesh width $\Delta x$, we seek entropy variables

$$
\begin{aligned}
\mathbf{V}^{\Delta x} \in \mathcal{V}_{p} & =\left(\mathbb{P}_{p}(\Omega \times[0, T])\right)^{m} \\
& =\left\{\mathbf{W} \in\left(L^{1}(\Omega \times[0, T])\right)^{m}:\left.\mathbf{W}\right|_{K \times I^{n}} \text { is a polynomial of degree } p \text { in each component }\right\}
\end{aligned}
$$

such that the following quasilinear variational form is satisfied for each $\mathbf{W}^{\Delta x} \in \mathcal{V}_{p}$ :

$$
\mathcal{B}\left(\mathbf{V}^{\Delta x}, \mathbf{W}^{\Delta x}\right):=\mathcal{B}_{\mathrm{DG}}\left(\mathbf{V}^{\Delta x}, \mathbf{W}^{\Delta x}\right)+\mathcal{B}_{\mathrm{SD}}\left(\mathbf{V}^{\Delta x}, \mathbf{W}^{\Delta x}\right)+\mathcal{B}_{\mathrm{SC}}\left(\mathbf{V}^{\Delta x}, \mathbf{W}^{\Delta x}\right)=0
$$


It consists of three parts, which we will describe in the following.

\subsection{The DG quasilinear form}

Following [9], the DG form for non-conservative systems is based on fluctuations rather than on numerical fluxes: a first-order path-conservative method for (1.1) is given by

$$
\mathbf{U}_{K}^{n+1}=\mathbf{U}_{K}^{n}-\frac{\Delta t^{n}}{|K|} \sum_{K^{\prime} \in \mathcal{N}(K)}\left|\partial_{K, K^{\prime}}\right| \mathbf{D}^{-}\left(\mathbf{U}_{K}^{n}, \mathbf{U}_{K^{\prime}}^{n} ; \nu_{K K^{\prime}}\right)
$$

Here $\mathbf{U}_{K}^{n}$ is an approximation of the average of the solution at the element $K$ at time $t^{n},|K|$ and $\left|\partial_{K, K^{\prime}}\right|$ represent the Lebesgue measures of the element $K$ in $\mathbb{R}^{d}$ and of its intersection with $K^{\prime}$ in $\mathbb{R}^{d-1}$. The so-called fluctuations $\mathbf{D}^{-}$satisfy

$$
\mathbf{D}^{-}(\mathbf{U}, \mathbf{U} ; \nu)=0
$$

and the path consistency condition

$$
\mathbf{D}^{-}\left(\mathbf{U}_{-}, \mathbf{U}_{+} ; \nu\right)+\mathbf{D}^{+}\left(\mathbf{U}_{-}, \mathbf{U}_{+} ; \nu\right)=\int_{0}^{1} \sum_{k=1}^{d} A^{k}\left(\Phi\left(s ; \mathbf{U}_{-}, \mathbf{U}_{+}\right)\right) \partial_{s} \Phi\left(s ; \mathbf{U}_{-}, \mathbf{U}_{+}\right) \nu^{k} \mathrm{~d} s,
$$

where $\Phi:[0,1] \times \mathbb{R}^{m} \times \mathbb{R}^{m} \rightarrow \mathbb{R}^{m}$ is the chosen family of paths, with the definition

$$
\mathbf{D}^{+}\left(\mathbf{U}_{-}, \mathbf{U}_{+} ; \nu\right)=\mathbf{D}^{-}\left(\mathbf{U}_{+}, \mathbf{U}_{-} ;-\nu\right)
$$

Notice that (2.5) is a formal consistency condition related to the chosen family of paths.

The fluctuations are assumed to be functions of the entropy variables and to satisfy:

$$
\left\langle\mathbf{V}_{-}, \mathbf{D}^{-}\left(\mathbf{V}_{-}, \mathbf{V}_{+} ; \nu\right)\right\rangle+\left\langle\mathbf{V}_{+}, \mathbf{D}^{+}\left(\mathbf{V}_{-}, \mathbf{V}_{+} ; \nu\right)\right\rangle=\sum_{k=1}^{d}\left(Q^{k}\left(\mathbf{V}_{+}\right)-Q^{k}\left(\mathbf{V}_{-}\right)\right) \nu^{k}
$$

where the notation

$$
\mathbf{D}^{ \pm}\left(\mathbf{V}_{-}, \mathbf{V}_{+} ; \nu\right)=\mathbf{D}^{ \pm}\left(\mathbf{U}\left(\mathbf{V}_{-}\right), \mathbf{U}\left(\mathbf{V}_{+}\right) ; \nu\right), \quad Q^{k}\left(\mathbf{V}_{ \pm}\right)=Q^{k}\left(\mathbf{U}\left(\mathbf{V}_{ \pm}\right)\right)
$$

has been used for simplicity. This is the key requirement on the form of the fluctuations that will enable us to prove the entropy stability. In [9] the existence of fluctuations satisfying (2.7) for any family of paths has been shown.

The condition (2.7) will not lead to a sufficient amount of numerical diffusion. Therefore, the fluctuations are supplemented with a diffusion operator

$$
\mathbb{D}\left(\mathbf{V}_{-}, \mathbf{V}_{+} ; \nu\right)=\mathbf{D}^{-}\left(\mathbf{V}_{-}, \mathbf{V}_{+} ; \nu\right)-\frac{1}{2} \mathbf{R}\left(\mathbf{V}_{-}, \mathbf{V}_{+} ; \nu\right)\left(\mathbf{V}_{+}-\mathbf{V}_{-}\right)
$$

where the numerical viscosity matrix $\mathbf{R}\left(\mathbf{V}_{-}, \mathbf{V}_{+} ; \nu\right)$ is assumed to be positive and to satisfy the symmetry property

$$
\mathbf{R}\left(\mathbf{V}_{-}, \mathbf{V}_{+} ; \nu\right)=\mathbf{R}\left(\mathbf{V}_{+}, \mathbf{V}_{-} ;-\nu\right)
$$


For instance, a Rusanov type diffusion term can be used, which is given by

$$
\mathbf{R}\left(\mathbf{V}_{-}, \mathbf{V}_{+} ; \nu\right)=\epsilon \max \left\{\left|\lambda_{\max }\left(\mathbf{V}_{-} ; \nu\right)\right|,\left|\lambda_{\max }\left(\mathbf{V}_{+} ; \nu\right)\right|\right\} \mathbf{U}_{\mathbf{V}}(\overline{\mathbf{V}})
$$

where $\lambda_{\max }(\mathbf{U} ; \nu)$ is the maximal wave speed in the direction of $\nu$, i.e. the largest (in amplitude) eigenvector of the matrix:

$$
A(\mathbf{U}, \nu)=\sum_{k=1}^{d} \nu_{k} A^{k}(\mathbf{U}) .
$$

The form $\mathcal{B}_{\mathrm{DG}}$ is then given by

$$
\begin{aligned}
\mathcal{B}_{\mathrm{DG}}\left(\mathbf{V}^{\Delta x}, \mathbf{W}^{\Delta x}\right)= & \sum_{n, K} \int_{I^{n}} \int_{K}\left(-\left\langle\mathbf{U}\left(\mathbf{V}^{\Delta x}\right), \mathbf{W}_{t}^{\Delta x}\right\rangle+\sum_{k=1}^{d}\left\langle A^{k}\left(\mathbf{V}^{\Delta x}\right) \mathbf{V}_{x_{k}}^{\Delta x}, \mathbf{W}^{\Delta x}\right\rangle\right) \mathrm{d} x \mathrm{~d} t \\
& +\sum_{n, K} \int_{K}\left\langle\mathbb{U}\left(\mathbf{V}_{n+1,-}^{\Delta x}, \mathbf{V}_{n+1,+}^{\Delta x}\right), \mathbf{W}_{n+1,-}^{\Delta x}\right\rangle \mathrm{d} x-\sum_{n, K} \int_{K}\left\langle\mathbb{U}\left(\mathbf{V}_{n,-}^{\Delta x}, \mathbf{V}_{n,+}^{\Delta x}\right), \mathbf{W}_{n,+}^{\Delta x}\right\rangle \mathrm{d} x \\
& +\sum_{n, K} \sum_{K^{\prime} \in \mathcal{N}(K)} \int_{I^{n}} \int_{\partial_{K K^{\prime}}}\left\langle\mathbb{D}\left(\mathbf{V}_{K,-}^{\Delta x}, \mathbf{V}_{K,+}^{\Delta x} ; \nu_{K K^{\prime}}\right), \mathbf{W}_{K,-}^{\Delta x}\right\rangle \mathrm{d} \sigma(x) \mathrm{d} t .
\end{aligned}
$$

It can be obtained as follows: multiply the non-conservative system (1.1) with a test function, integrate over space and time, and perform an integration by parts in the temporal direction. The fluxes at the temporal element boundary are replaced by numerical fluxes $\mathbb{U}$, and at the spatial element boundaries, the fluctuations $\mathbb{D}$ are introduced to take into account the discontinuities of the test functions: notice that, according to (2.4), they vanish when the functions are continuous at the edge of an element.

Upwind fluxes are used for the temporal numerical fluxes

$$
\mathbb{U}\left(\mathbf{V}_{-}, \mathbf{V}_{+}\right)=\mathbf{U}\left(\mathbf{V}_{-}\right) \text {. }
$$

This ensures causality and allows us to march in time.

Inserting the numerical fluxes and the fluctuations into the form $\mathcal{B}_{\mathrm{DG}}(2.12)$, we obtain

$$
\begin{aligned}
\mathcal{B}_{\mathrm{DG}}\left(\mathbf{V}^{\Delta x}, \mathbf{W}^{\Delta x}\right)= & \sum_{n, K} \int_{I^{n}} \int_{K}\left(-\left\langle\mathbf{U}\left(\mathbf{V}^{\Delta x}\right), \mathbf{W}_{t}^{\Delta x}\right\rangle+\sum_{k=1}^{d}\left\langle A^{k}\left(\mathbf{V}^{\Delta x}\right) \mathbf{V}_{x_{k}}^{\Delta x}, \mathbf{W}^{\Delta x}\right\rangle\right) \mathrm{d} x \mathrm{~d} t \\
& +\sum_{n, K} \int_{K}\left\langle\mathbf{U}\left(\mathbf{V}_{n+1,-}^{\Delta x}\right), \mathbf{W}_{n+1,-}^{\Delta x}\right\rangle \mathrm{d} x-\sum_{n, K} \int_{K}\left\langle\mathbf{U}\left(\mathbf{V}_{n,-}^{\Delta x}\right), \mathbf{W}_{n,+}^{\Delta x}\right\rangle \mathrm{d} x \\
& +\sum_{n, K} \sum_{K^{\prime} \in \mathcal{N}(K)} \int_{I^{n}} \int_{\partial_{K K^{\prime}}}\left(\left\langle\mathbf{D}^{-}\left(\mathbf{V}_{K,-}^{\Delta x}, \mathbf{V}_{K,+}^{\Delta x} ; \nu_{K K^{\prime}}^{k}\right), \mathbf{W}_{K,-}^{\Delta x}\right\rangle\right) \mathrm{d} \sigma(x) \mathrm{d} t \\
& -\frac{1}{2} \sum_{n, K} \sum_{K^{\prime} \in \mathcal{N}(K)} \int_{I^{n}} \int_{\partial_{K K^{\prime}}}\left\langle\hat{\mathbf{R}}\left(\mathbf{V}_{K,+}^{\Delta x}-\mathbf{V}_{K,-}^{\Delta x}\right), \mathbf{W}_{K,-}^{\Delta x}\right\rangle \mathrm{d} \sigma(x) \mathrm{d} t,
\end{aligned}
$$

where

$$
\hat{\mathbf{R}}=\mathbf{R}\left(\mathbf{V}_{-}, \mathbf{V}_{+} ; \nu\right) .
$$


Remark 2.1. If this method is applied to a system which is actually conservative, i.e. $A^{k}(\mathbf{V})=\mathbf{F}_{\mathbf{V}}^{k}(\mathbf{V})$, the DG form reduces to the one presented in $[17,18]$ for conservation laws (with a potentially different entropyconservative flux). Indeed, the consistency condition (2.5) leads to the equality

$$
\mathbf{D}^{-}\left(\mathbf{U}_{-}, \mathbf{U}_{+} ; \nu\right)+\mathbf{D}^{+}\left(\mathbf{U}_{-}, \mathbf{U}_{+} ; \nu\right)=\mathbf{F}_{\nu}\left(\mathbf{U}_{+}\right)-\mathbf{F}_{\nu}\left(\mathbf{U}_{-}\right)
$$

where

$$
\mathbf{F}_{\nu}(\mathbf{U})=\sum_{k=1}^{d} \mathbf{F}^{k}(\mathbf{U}) \nu^{k}
$$

Then, if we define

$$
\mathbb{F}^{*}\left(\mathbf{U}_{-}, \mathbf{U}_{+} ; \nu\right)=\mathbf{F}_{\nu}\left(\mathbf{U}_{+}\right)-\mathbf{D}^{+}\left(\mathbf{U}_{-}, \mathbf{U}_{+} ; \nu\right)
$$

or, equivalently,

$$
\mathbb{F}^{*}\left(\mathbf{U}_{-}, \mathbf{U}_{+} ; \nu\right)=\mathbf{D}^{-}\left(\mathbf{U}_{-}, \mathbf{U}_{+} ; \nu\right)+\mathbf{F}_{\nu}\left(\mathbf{U}_{-}\right),
$$

then $\mathbb{F}^{*}$ is a consistent numerical flux due to condition (2.4). Moreover, condition (2.7) implies that this is an entropy-conservative numerical flux (see [9]). Using (2.16) and integration by parts in (2.14), the form $\mathcal{B}_{\mathrm{DG}}$ can be written in the conservative form

$$
\begin{aligned}
\mathcal{B}_{\mathrm{DG}}\left(\mathbf{V}^{\Delta x}, \mathbf{W}^{\Delta x}\right)= & -\sum_{n, K} \int_{I^{n}} \int_{K}\left(\left\langle\mathbf{U}\left(\mathbf{V}^{\Delta x}\right), \mathbf{W}_{t}^{\Delta x}\right\rangle+\sum_{k=1}^{d}\left\langle\mathbf{F}^{k}\left(\mathbf{V}^{\Delta x}\right), \mathbf{W}_{x_{k}}^{\Delta x}\right\rangle\right) \mathrm{d} x \mathrm{~d} t \\
& +\sum_{n, K} \int_{K}\left\langle\mathbf{U}\left(\mathbf{V}_{n+1,-}^{\Delta x}\right), \mathbf{W}_{n+1,-}^{\Delta x}\right\rangle \mathrm{d} x-\sum_{n, K} \int_{K}\left\langle\mathbf{U}\left(\mathbf{V}_{n,-}^{\Delta x}\right), \mathbf{W}_{n,+}^{\Delta x}\right\rangle \mathrm{d} x \\
& +\sum_{n, K} \sum_{K^{\prime} \in \mathcal{N}(K)} \int_{I^{n}} \int_{\partial_{K K^{\prime}}}\left\langle\mathbb{F}^{*}\left(\mathbf{V}_{K,-}^{\Delta x}, \mathbf{V}_{K,+}^{\Delta x} ; \nu_{K K^{\prime}}\right), \mathbf{W}_{K,-}^{\Delta x}\right\rangle \mathrm{d} \sigma(x) \mathrm{d} t \\
& -\frac{1}{2} \sum_{n, K} \sum_{K^{\prime} \in \mathcal{N}(K)} \int_{I^{n}} \int_{\partial_{K K^{\prime}}}\left\langle\hat{\mathbf{R}}\left(\mathbf{V}_{K,+}^{\Delta x}-\mathbf{V}_{K,-}^{\Delta x}\right), \mathbf{W}_{K,-}^{\Delta x}\right\rangle \mathrm{d} \sigma(x) \mathrm{d} t,
\end{aligned}
$$

where notation $(2.8)$ and

$$
\mathbf{F}^{k}(\mathbf{V})=\mathbf{F}^{k}(\mathbf{U}(\mathbf{V})), \quad \mathbb{F}^{*}\left(\mathbf{V}_{-}, \mathbf{V}_{+} ; \nu\right)=\mathbb{F}^{*}\left(\mathbf{U}\left(\mathbf{V}_{-}\right), \mathbf{U}\left(\mathbf{V}_{+}\right) ; \nu\right),
$$

have been used.

\subsection{Streamline diffusion operator}

The DG formulation so far only adds diffusion across the boundary of each space-time element. There is yet no stabilizing diffusion inside each element in order to damp down unphysical intra-element oscillations. Therefore, a streamline diffusion operator is added in [17], which can easily be adapted to non-conservative systems. The equation residual (or intra-element residual) is now

$$
\operatorname{Res}:=\mathbf{U}\left(\mathbf{V}^{\Delta x}\right)_{t}+\sum_{k=1}^{d} A^{k}\left(\mathbf{V}^{\Delta x}\right) \mathbf{V}_{x_{k}}^{\Delta x}
$$


The streamline diffusion operator is then given by

$$
\mathcal{B}_{\mathrm{SD}}\left(\mathbf{V}^{\Delta x}, \mathbf{W}^{\Delta x}\right)=\sum_{n, K} \int_{I^{n}} \int_{K}\left\langle\left(\mathbf{U}_{\mathbf{V}}\left(\mathbf{V}^{\Delta x}\right) \mathbf{W}_{t}^{\Delta x}+\sum_{k=1}^{d} A^{k}\left(\mathbf{V}^{\Delta x}\right) \mathbf{W}_{x_{k}}^{\Delta x}\right), \mathbf{D}^{\mathrm{SD}} \operatorname{Res}\right\rangle \mathrm{d} x \mathrm{~d} t .
$$

The only difference with the conservative case is that, here, the flux Jacobians are replaced by the matrices $A^{k}$. The scaling matrix

$$
\mathbf{D}^{\mathrm{SD}}:=C^{\mathrm{SD}} \Delta t^{n} \mathbf{U}_{\mathbf{V}}^{-1}\left(\mathbf{V}^{\Delta x}\right)
$$

with a positive constant $C^{\mathrm{SD}}$ remains unchanged.

\subsection{Shock-capturing operator}

The experience in the case of equations in the conservation form (1.3) shows that streamline diffusion does not suffice in controlling intra-cell oscillations: see [17] and references therein. Therefore, an additional shock capturing operator is employed, which is similar to the one in [2], and can be easily adapted from the one in [17]. It is given by

$$
\mathcal{B}_{\mathrm{SC}}\left(\mathbf{V}^{\Delta x}, \mathbf{W}^{\Delta x}\right)=\sum_{n, K} \int_{I^{n}} \int_{K} D_{n, K}^{\mathrm{SC}}\left(\left\langle\mathbf{W}_{t}^{\Delta x}, \widetilde{\mathbf{U}_{\mathbf{V}}} \mathbf{V}_{t}^{\Delta x}\right\rangle+\sum_{k=1}^{d} \frac{\Delta x_{K}^{2}}{\left(\Delta t^{n}\right)^{2}}\left\langle\mathbf{W}_{x_{k}}^{\Delta x}, \widetilde{\mathbf{U}_{\mathbf{V}}} \mathbf{V}_{x_{k}}^{\Delta x}\right\rangle\right) \mathrm{d} x \mathrm{~d} t
$$

with $\widetilde{\mathbf{U}_{\mathbf{V}}}=\mathbf{U}_{\mathbf{V}}\left(\widetilde{\mathbf{V}}_{n, K}\right)$ for brevity and

$$
\widetilde{\mathbf{V}}_{n, K}=\frac{1}{\operatorname{meas}\left(I^{n} \times K\right)} \int_{I^{n}} \int_{K} \mathbf{V}^{\Delta x}(x, t) \mathrm{d} x \mathrm{~d} t,
$$

being the cell average. The scaling factor is

$$
D_{n, K}^{\mathrm{SC}}=\frac{\Delta t^{n} C^{\mathrm{SC}} \overline{\operatorname{Res}}_{n, K}+\left(\Delta t^{n}\right)^{\frac{1}{2}} \bar{C}^{\mathrm{SC}} \overline{\operatorname{BRes}}_{n, K}}{\sqrt{\int_{I^{n}} \int_{K}\left(\left\langle\mathbf{V}_{t}^{\Delta x}, \widetilde{\mathbf{U}_{\mathbf{V}}} \mathbf{V}_{t}^{\Delta x}\right\rangle+\sum_{k=1}^{d} \frac{\Delta x_{K}{ }^{2}}{\left(\Delta t^{n}\right)^{2}}\left\langle\mathbf{V}_{x_{k}}^{\Delta x}, \widetilde{\mathbf{U}_{\mathbf{V}}} \mathbf{V}_{x_{k}}^{\Delta x}\right\rangle\right) \mathrm{d} x \mathrm{~d} t+\bar{\epsilon}}},
$$

with $\bar{\epsilon}:=|K|^{\frac{1}{2}}\left(\Delta t^{n}\right)^{\frac{-1}{2}}\left(\frac{\Delta x}{\operatorname{diam}(\Omega)}\right)^{\theta}$ and $\theta \geq 1 / 2$ (chosen as 1). It relies on the integrated intra-element residual

$$
\overline{\operatorname{Res}}_{n, K}:=\sqrt{\int_{I^{n}} \int_{K}\left\langle\operatorname{Res}, \mathbf{U}_{\mathbf{V}}^{-1}\left(\mathbf{V}^{\Delta x}\right) \operatorname{Res}\right\rangle \mathrm{d} x \mathrm{~d} t},
$$

and on the integrated boundary residual

$$
\begin{aligned}
\overline{\operatorname{BRes}}_{n, K}:= & \left(\int_{K}\left\|\mathbf{U}\left(\mathbf{V}_{n,-}^{\Delta x}\right)-\mathbf{U}\left(\mathbf{V}_{n,+}^{\Delta x}\right)\right\|_{\mathbf{U}_{\mathbf{V}}^{-1}\left(\mathbf{V}_{n,+}^{\Delta x}\right)}^{2} \mathrm{~d} x\right. \\
& \left.+\sum_{K^{\prime}} \int_{I^{n}} \int_{\partial_{K K^{\prime}}} \frac{\Delta t^{n}}{\Delta x_{K}}\left\|\mathbb{D}\left(\mathbf{V}_{K,-}^{\Delta x}, \mathbf{V}_{K,+}^{\Delta x} ; \nu_{K K^{\prime}}\right)\right\|_{\mathbf{U}_{\mathbf{V}}^{-1}\left(\mathbf{V}_{K,-}^{\Delta x}\right)}^{2} \mathrm{~d} \sigma \mathrm{d} t\right)^{\frac{1}{2}} .
\end{aligned}
$$




\section{ENTROPy STABILITY}

We consider the entropy stability of the shock-capturing streamline diffusion space-time DG scheme below. Although the proof follows the steps of the corresponding results for systems of conservation laws in [18], it is fully developed here for the sake of completeness.

Theorem 3.1. Consider the non-conservative system (1.1) with a uniformly convex entropy function $S$ and entropy flux functions $Q^{k}(1 \leq k \leq d)$. For simplicity, assume that the exact and approximate solutions have compact support inside the spatial domain $\Omega$. Let the final time be denoted by $t^{N}$. Then, the streamline diffusion shock-capturing discontinuous Galerkin scheme (2.2) approximating (1.1) is entropy-stable, i.e. the approximate solutions $\mathbf{U}^{\Delta x}=\mathbf{U}\left(\mathbf{V}^{\Delta x}\right)$ satisfy

$$
\int_{\Omega} S\left(\mathbf{U}^{\Delta x}\left(x, t_{-}^{N}\right)\right) \mathrm{d} x \leq \int_{\Omega} S\left(\mathbf{U}^{\Delta x}\left(x, t_{-}^{0}\right)\right) \mathrm{d} x .
$$

Proof. To prove entropy stability, we proceed to show a series of claims.

Claim 1: The streamline diffusion operator (2.18) is positive, i.e.

$$
\mathcal{B}_{\mathrm{SD}}\left(\mathbf{V}^{\Delta x}, \mathbf{V}^{\Delta x}\right) \geq 0
$$

Setting $\mathbf{W}^{\Delta x}=\mathbf{V}^{\Delta x}$ in (2.18) (notice that $\mathbf{V}^{\Delta x} \in \mathcal{V}_{p}$ and thus it is an admissible test function) we obtain

$$
\begin{aligned}
\mathcal{B}_{\mathrm{SD}}\left(\mathbf{V}^{\Delta x}, \mathbf{V}^{\Delta x}\right) & =\sum_{n, K} \int_{I^{n}} \int_{K}\left\langle\left(\mathbf{U}_{\mathbf{V}}\left(\mathbf{V}^{\Delta x}\right) \mathbf{V}_{t}^{\Delta x}+\sum_{k=1}^{d} A^{k}\left(\mathbf{V}^{\Delta x}\right) \mathbf{V}_{x_{k}}^{\Delta x}\right), \mathbf{D}^{\mathrm{SD}} \operatorname{Res}\right\rangle \mathrm{d} x \mathrm{~d} t \\
& =\sum_{n, K} \int_{I^{n}} \int_{K}\left\langle\left(\mathbf{U}\left(\mathbf{V}^{\Delta x}\right)_{t}+\sum_{k=1}^{d} A^{k}\left(\mathbf{V}^{\Delta x}\right) \mathbf{V}_{x_{k}}^{\Delta x}\right), \mathbf{D}^{\mathrm{SD}} \operatorname{Res}\right\rangle \mathrm{d} x \mathrm{~d} t \\
& =\sum_{n, K} \int_{I^{n}} \int_{K}\left\langle\operatorname{Res}, \mathbf{D}^{\mathrm{SD}} \operatorname{Res}\right\rangle \mathrm{d} x \mathrm{~d} t \quad(\text { from }(2.17)), \\
& \geq C C^{\mathrm{SD}} \Delta x \sum_{n, K} \int_{I^{n}} \int_{K}\|\operatorname{Res}\|^{2} \mathrm{~d} x \mathrm{~d} t \\
& \geq 0 .
\end{aligned}
$$

Claim 2: The shock-capturing operator (2.20) is positive, i.e.:

$$
\mathcal{B}_{\mathrm{SC}}\left(\mathbf{V}^{\Delta x}, \mathbf{V}^{\Delta x}\right) \geq 0
$$

First, we observe that the uniform convexity of the entropy function $S$ implies that the matrices $\mathbf{U}_{\mathbf{V}}$ and $\mathbf{U}_{\mathbf{V}}^{-1}$ are positive definite. This implies that the term $D_{n, K}^{\mathrm{SC}}$ is real and positive.

We set as test function $\mathbf{W}^{\Delta x}=\mathbf{V}^{\Delta x}$ in (2.20a) and obtain

$$
\begin{aligned}
\mathcal{B}_{\mathrm{SC}}\left(\mathbf{V}^{\Delta x}, \mathbf{V}^{\Delta x}\right)= & \sum_{n, K} \int_{I^{n}} \int_{K} D_{n, K}^{\mathrm{SC}}\left(\left\langle\mathbf{V}_{t}^{\Delta x}, \widetilde{\mathbf{U}_{\mathbf{V}}} \mathbf{V}_{t}^{\Delta x}\right\rangle+\sum_{k=1}^{d} \frac{\Delta x_{K}^{2}}{\left(\Delta t^{n}\right)^{2}}\left\langle\mathbf{V}_{x_{k}}^{\Delta x}, \widetilde{\mathbf{U}_{\mathbf{V}}} \mathbf{V}_{x_{k}}^{\Delta x}\right\rangle\right) \mathrm{d} x \mathrm{~d} t \\
& \geq \sum_{n, K} \lambda_{1} D_{n, K}^{\mathrm{SC}} \int_{I^{n}} \int_{K}\left\|\nabla_{x t} \mathbf{V}^{\Delta x}\right\|^{2} \mathrm{~d} x \mathrm{~d} t \\
& \geq 0 .
\end{aligned}
$$


Here, $\lambda_{1}$ is the smallest eigenvalue of the positive definite matrix $\mathbf{U}_{\mathbf{V}}$. If $\mathbf{U}_{\mathbf{V}}$ is uniformly positive definite, one obtains $\lambda_{1}>0$.

Claim 3: Define the spatial part of the DG form $\mathcal{B}_{\mathrm{DG}}(2.14)$ as

$$
\begin{aligned}
\mathcal{B}_{\mathrm{DG}}^{s}\left(\mathbf{V}^{\Delta x}, \mathbf{W}^{\Delta x}\right)= & \sum_{n, K} \int_{I^{n}} \int_{K} \sum_{k=1}^{d}\left\langle A^{k}\left(\mathbf{V}^{\Delta x}\right) \mathbf{V}_{x_{k}}^{\Delta x}, \mathbf{W}^{\Delta x}\right\rangle \mathrm{d} x \mathrm{~d} t \\
& +\sum_{n, K, K^{\prime}} \int_{I^{n}} \int_{\partial_{K K^{\prime}}}\left\langle\mathbf{D}^{-}\left(\mathbf{V}_{K,-}^{\Delta x}, \mathbf{V}_{K,+}^{\Delta x} ; \nu_{K K^{\prime}}\right), \mathbf{W}_{K,-}^{\Delta x}\right\rangle \mathrm{d} \sigma \mathrm{d} t \\
& -\frac{1}{2} \sum_{n, K, K^{\prime}} \int_{I^{n}} \int_{\partial_{K K^{\prime}}}\left\langle\mathbf{W}_{K,-}^{\Delta x}, \hat{\mathbf{R}}\left(\mathbf{V}_{K,+}^{\Delta x}-\mathbf{V}_{K,-}^{\Delta x}\right)\right\rangle \mathrm{d} \sigma \mathrm{d} t .
\end{aligned}
$$

We claim that

$$
\mathcal{B}_{\mathrm{DG}}^{s}\left(\mathbf{V}^{\Delta x}, \mathbf{V}^{\Delta x}\right) \geq 0
$$

From the compatibility of the entropy flux (1.4), we obtain

$$
\left\langle A^{k}(\mathbf{V}) \mathbf{V}_{x_{k}}, \mathbf{V}\right\rangle=Q^{k}(\mathbf{V})_{\mathbf{V}} \mathbf{V}_{x_{k}}=Q^{k}(\mathbf{V})_{x_{k}}, \quad k=1, \ldots, d
$$

Therefore,

$$
\begin{aligned}
\sum_{n, K} \int_{I^{n}} \int_{K} \sum_{k=1}^{d}\left\langle A^{k}\left(\mathbf{V}^{\Delta x}\right) \mathbf{V}_{x_{k}}^{\Delta x}, \mathbf{V}^{\Delta x}\right\rangle \mathrm{d} x \mathrm{~d} t & =\sum_{n, K} \int_{I^{n}} \int_{K} \sum_{k=1}^{d} Q^{k}\left(\mathbf{V}^{\Delta x}\right)_{x_{k}} \mathrm{~d} x \mathrm{~d} t \\
& =\sum_{n, K, K^{\prime}} \int_{I^{n}} \int_{\partial_{K K^{\prime}}} \sum_{k=1}^{d} Q^{k}\left(\mathbf{V}_{K,-}^{\Delta x}\right) \nu_{K K^{\prime}}^{k} \mathrm{~d} \sigma \mathrm{d} t .
\end{aligned}
$$

Using the above identities, we obtain

$$
\begin{aligned}
\mathcal{B}_{\mathrm{DG}}^{s}\left(\mathbf{V}^{\Delta x}, \mathbf{V}^{\Delta x}\right)= & \sum_{n, K} \int_{I^{n}} \int_{K} \sum_{k=1}^{d}\left\langle A^{k}\left(\mathbf{V}^{\Delta x}\right) \mathbf{V}_{x_{k}}^{\Delta x}, \mathbf{V}^{\Delta x}\right\rangle \mathrm{d} x \mathrm{~d} t \\
& +\sum_{n, K, K^{\prime}} \int_{I^{n}} \int_{\partial_{K K^{\prime}}}\left\langle\mathbf{D}^{-}\left(\mathbf{V}_{K,-}^{\Delta x}, \mathbf{V}_{K,+}^{\Delta x} ; \nu_{K K^{\prime}}\right), \mathbf{V}_{K,-}^{\Delta x}\right\rangle \mathrm{d} \sigma \mathrm{d} t \\
& -\frac{1}{2} \sum_{n, K, K^{\prime}} \int_{I^{n}} \int_{\partial_{K K^{\prime}}}\left\langle\mathbf{V}_{K,-}^{\Delta x}, \hat{\mathbf{R}}\left(\mathbf{V}_{K,+}^{\Delta x}-\mathbf{V}_{K,-}^{\Delta x}\right)\right\rangle \mathrm{d} \sigma \mathrm{d} t \\
= & \sum_{n, K, K^{\prime}} \int_{I^{n}} \int_{\partial_{K K^{\prime}}}\left(\left\langle\mathbf{D}^{-}\left(\mathbf{V}_{K,-}^{\Delta x}, \mathbf{V}_{K,+}^{\Delta x} ; \nu_{K K^{\prime}}\right), \mathbf{V}_{K,-}^{\Delta x}\right\rangle+\sum_{k=1}^{d} Q^{k}\left(\mathbf{V}_{K,-}^{\Delta x}\right) \nu_{K K^{\prime}}^{k}\right) \mathrm{d} \sigma \mathrm{d} t \\
& -\frac{1}{2} \sum_{n, K, K^{\prime}} \int_{I^{n}} \int_{\partial_{K K^{\prime}}}\left\langle\mathbf{V}_{K,-}^{\Delta x}, \hat{\mathbf{R}}\left(\mathbf{V}_{K,+}^{\Delta x}-\mathbf{V}_{K,-}^{\Delta x}\right)\right\rangle \mathrm{d} \sigma \mathrm{d} t \\
= & \frac{1}{2} \sum_{n, K, K^{\prime}} \int_{I^{n}} \int_{\partial_{K K^{\prime}}}\left(\left\langle\mathbf{D}^{-}\left(\mathbf{V}_{K,-}^{\Delta x}, \mathbf{V}_{K,+}^{\Delta x} ; \nu_{K K^{\prime}}\right), \mathbf{V}_{K,-}^{\Delta x}\right\rangle+\sum_{k=1}^{d} Q^{k}\left(\mathbf{V}_{K,-}^{\Delta x}\right) \nu_{K K^{\prime}}^{k}\right) \mathrm{d} \sigma \mathrm{d} t
\end{aligned}
$$




$$
\begin{aligned}
& +\frac{1}{2} \sum_{n, K, K^{\prime}} \int_{I^{n}} \int_{\partial_{K K^{\prime}}}\left(\left\langle\mathbf{D}^{-}\left(\mathbf{V}_{K,-}^{\Delta x}, \mathbf{V}_{K,+}^{\Delta x} ; \nu_{K K^{\prime}}\right), \mathbf{V}_{K,-}^{\Delta x}\right\rangle+\sum_{k=1}^{d} Q^{k}\left(\mathbf{V}_{K,-}^{\Delta x}\right) \nu_{K K^{\prime}}^{k}\right) \mathrm{d} \sigma \mathrm{d} t \\
& -\frac{1}{4} \sum_{n, K, K^{\prime}} \int_{I^{n}} \int_{\partial_{K K^{\prime}}}\left\langle\mathbf{V}_{K,-}^{\Delta x}, \hat{\mathbf{R}}\left(\mathbf{V}_{K,+}^{\Delta x}-\mathbf{V}_{K,-}^{\Delta x}\right)\right\rangle \mathrm{d} \sigma \mathrm{d} t \\
& -\frac{1}{4} \sum_{n, K, K^{\prime}} \int_{I^{n}} \int_{\partial_{K K^{\prime}}}\left\langle\mathbf{V}_{K,-}^{\Delta x}, \hat{\mathbf{R}}\left(\mathbf{V}_{K,+}^{\Delta x}-\mathbf{V}_{K,-}^{\Delta x}\right)\right\rangle \mathrm{d} \sigma \mathrm{d} t
\end{aligned}
$$

Changing the roles of $K$ and $K^{\prime}$ in the second and fourth summands and rewriting the arguments of the sums, the above expression reduces to

$$
\begin{aligned}
& \mathcal{B}_{\mathrm{DG}}^{s}\left(\mathbf{V}^{\Delta x}, \mathbf{V}^{\Delta x}\right) \\
& =\frac{1}{2} \sum_{n, K, K^{\prime}} \int_{I^{n}} \int_{\partial_{K K^{\prime}}}\left(\left\langle\mathbf{D}^{-}\left(\mathbf{V}_{K,-}^{\Delta x}, \mathbf{V}_{K,+}^{\Delta x} ; \nu_{K K^{\prime}}\right), \mathbf{V}_{K,-}^{\Delta x}\right\rangle+\left\langle\mathbf{D}^{+}\left(\mathbf{V}_{K,-}^{\Delta x}, \mathbf{V}_{K,+}^{\Delta x} ; \nu_{K K^{\prime}}\right), \mathbf{V}_{K,+}^{\Delta x}\right\rangle\right. \\
& \underbrace{\left.-\sum_{k=1}^{d}\left(Q^{k}\left(\mathbf{V}_{K,+}^{\Delta x}\right)-Q^{k}\left(\mathbf{V}_{K,-}^{\Delta x}\right)\right) \nu_{K K^{\prime}}^{k}\right)}_{\text {from }(2.7)} \mathrm{d} \sigma \mathrm{d} t \\
& +\frac{1}{4} \sum_{n, K, K^{\prime}} \int_{I^{n}} \int_{\partial_{K K^{\prime}}}\left\langle\mathbf{V}_{K,+}^{\Delta x}-\mathbf{V}_{K,-}^{\Delta x}, \hat{\mathbf{R}}\left(\mathbf{V}_{K,+}^{\Delta x}-\mathbf{V}_{K,-}^{\Delta x}\right)\right\rangle \mathrm{d} \sigma \mathrm{d} t \\
& =\frac{1}{4} \sum_{n, K, K^{\prime}} \int_{I^{n}} \int_{\partial_{K K^{\prime}}}\left\langle\mathbf{V}_{K,+}^{\Delta x}-\mathbf{V}_{K,-}^{\Delta x}, \hat{\mathbf{R}}\left(\mathbf{V}_{K,+}^{\Delta x}-\mathbf{V}_{K,-}^{\Delta x}\right)\right\rangle \mathrm{d} \sigma \mathrm{d} t \\
& \geq 0 \quad \text { (as } \hat{\mathbf{R}} \text { is positive). }
\end{aligned}
$$

Claim 4: Define the temporal part of the DG form $\mathcal{B}_{\mathrm{DG}}(2.14)$ as

$$
\begin{aligned}
\mathcal{B}_{\mathrm{DG}}^{t}\left(\mathbf{V}^{\Delta x}, \mathbf{W}^{\Delta x}\right)= & -\sum_{n, K} \int_{I^{n}} \int_{K}\left\langle\mathbf{U}\left(\mathbf{V}^{\Delta x}\right), \mathbf{W}_{t}^{\Delta x}\right\rangle \mathrm{d} x \mathrm{~d} t \\
& +\sum_{n, K} \int_{K}\left\langle\mathbf{U}\left(\mathbf{V}_{n+1,-}^{\Delta x}\right), \mathbf{W}_{n+1,-}^{\Delta x}\right\rangle \mathrm{d} x-\sum_{n, K} \int_{K}\left\langle\mathbf{U}\left(\mathbf{V}_{n,-}^{\Delta x}\right), \mathbf{W}_{n,+}^{\Delta x}\right\rangle \mathrm{d} x .
\end{aligned}
$$

We claim that

$$
\mathcal{B}_{\mathrm{DG}}^{t}\left(\mathbf{V}^{\Delta x}, \mathbf{V}^{\Delta x}\right) \geq \int_{\Omega} S\left(\mathbf{U}\left(\mathbf{V}^{\Delta x}\left(x, t_{-}^{N}\right)\right)\right) \mathrm{d} x-\int_{\Omega} S\left(\mathbf{U}\left(\mathbf{V}^{\Delta x}\left(x, t_{-}^{0}\right)\right)\right) \mathrm{d} x .
$$

Setting $\mathbf{W}^{\Delta x}=\mathbf{V}^{\Delta x}$ in (3.10), we obtain

$$
\begin{aligned}
\mathcal{B}_{\mathrm{DG}}^{t}\left(\mathbf{V}^{\Delta x}, \mathbf{V}^{\Delta x}\right)= & -\sum_{n, K} \int_{I^{n}} \int_{K}\left\langle\mathbf{U}\left(\mathbf{V}^{\Delta x}\right), \mathbf{V}_{t}^{\Delta x}\right\rangle \mathrm{d} x \mathrm{~d} t \\
& +\sum_{n, K} \int_{K}\left\langle\mathbf{U}\left(\mathbf{V}_{n+1,-}^{\Delta x}\right), \mathbf{V}_{n+1,-}^{\Delta x}\right\rangle \mathrm{d} x-\sum_{n, K} \int_{K}\left\langle\mathbf{U}\left(\mathbf{V}_{n,-}^{\Delta x}\right), \mathbf{V}_{n,+}^{\Delta x}\right\rangle \mathrm{d} x \\
= & \sum_{n, K} \int_{I^{n}} \int_{K}\left\langle\mathbf{U}\left(\mathbf{V}^{\Delta x}\right)_{t}, \mathbf{V}^{\Delta x}\right\rangle \mathrm{d} x \mathrm{~d} t \quad \text { (integrating by parts) }
\end{aligned}
$$




$$
\begin{aligned}
& -\sum_{n, K} \int_{K}\left\langle\mathbf{U}\left(\mathbf{V}_{n+1,-}^{\Delta x}\right), \mathbf{V}_{n+1,-}^{\Delta x}\right\rangle \mathrm{d} x+\sum_{n, K} \int_{K}\left\langle\mathbf{U}\left(\mathbf{V}_{n,+}^{\Delta x}\right), \mathbf{V}_{n,+}^{\Delta x}\right\rangle \mathrm{d} x \\
& +\sum_{n, K} \int_{K}\left\langle\mathbf{U}\left(\mathbf{V}_{n+1,-}^{\Delta x}\right), \mathbf{V}_{n+1,-}^{\Delta x}\right\rangle \mathrm{d} x-\sum_{n, K} \int_{K}\left\langle\mathbf{U}\left(\mathbf{V}_{n,-}^{\Delta x}\right), \mathbf{V}_{n,+}^{\Delta x}\right\rangle \mathrm{d} x \\
= & \sum_{n, K} \int_{I^{n}} \int_{K} S\left(\mathbf{U}\left(\mathbf{V}^{\Delta x}\right)\right)_{t} \mathrm{~d} x \mathrm{~d} t \quad \text { (definition of entropy variables) } \\
& -\sum_{n, K} \int_{K}\left\langle\left(\mathbf{U}\left(\mathbf{V}_{n,-}^{\Delta x}\right)-\mathbf{U}\left(\mathbf{V}_{n,+}^{\Delta x}\right)\right), \mathbf{V}_{n,+}^{\Delta x}\right\rangle \mathrm{d} x \\
= & \sum_{n, K} \int_{K}\left(S\left(\mathbf{U}\left(\mathbf{V}_{n+1,-}^{\Delta x}\right)\right)-S\left(\mathbf{U}\left(\mathbf{V}_{n,-}^{\Delta x}\right)\right)\right) \mathrm{d} x \\
& +\sum_{n, K} \int_{K}\left(S\left(\mathbf{U}\left(\mathbf{V}_{n,-}^{\Delta x}\right)\right)-S\left(\mathbf{U}\left(\mathbf{V}_{n,+}^{\Delta x}\right)\right)\right) \mathrm{d} x \\
& -\sum_{n, K} \int_{K}\left\langle\left(\mathbf{U}\left(\mathbf{V}_{n,-}^{\Delta x}\right)-\mathbf{U}\left(\mathbf{V}_{n,+}^{\Delta x}\right)\right), \mathbf{V}_{n,+}^{\Delta x}\right\rangle \mathrm{d} x .
\end{aligned}
$$

Following [2, 28], we define $\mathbf{V}(\theta)=\mathbf{V}_{n,+}^{\Delta x}+\theta\left(\mathbf{V}_{n,-}^{\Delta x}-\mathbf{V}_{n,+}^{\Delta x}\right)=\theta \mathbf{V}_{n,-}^{\Delta x}+(1-\theta) \mathbf{V}_{n,+}^{\Delta x}$ and compute

$$
\begin{aligned}
S(\mathbf{U} & \left.\left(\mathbf{V}_{n,-}^{\Delta x}\right)\right)-S\left(\mathbf{U}\left(\mathbf{V}_{n,+}^{\Delta x}\right)\right) \\
& =\int_{0}^{1} \frac{d}{d \theta}(S(\mathbf{U}(\mathbf{V}(\theta)))) d \theta \\
& =\int_{0}^{1}\langle\underbrace{S_{\mathbf{U}}(\mathbf{U}(\mathbf{V}(\theta)))}_{\mathbf{V}(\theta)}, \mathbf{U}_{\mathbf{V}}(\theta) \underbrace{\mathbf{V}_{\theta}(\theta)}_{\mathbf{V}_{n,-}^{\Delta x}-\mathbf{V}_{n,+}^{\Delta x}}\rangle d \theta \\
& =\int_{0}^{1}\left\langle\mathbf{V}_{n,+}^{\Delta x}, \mathbf{U}_{\mathbf{V}}(\theta) \mathbf{V}_{\theta}(\theta)\right\rangle d \theta+\int_{0}^{1} \theta\left\langle\left(\mathbf{V}_{n,-}^{\Delta x}-\mathbf{V}_{n,+}^{\Delta x}\right), \mathbf{U}_{\mathbf{V}}(\theta)\left(\mathbf{V}_{n,-}^{\Delta x}-\mathbf{V}_{n,+}^{\Delta x}\right)\right\rangle d \theta \\
& =\left\langle\mathbf{V}_{n,+}^{\Delta x}, \mathbf{U}\left(\mathbf{V}_{n,-}^{\Delta x}\right)-\mathbf{U}\left(\mathbf{V}_{n,+}^{\Delta x}\right)\right\rangle+\int_{0}^{1} \theta\left\langle\left(\mathbf{V}_{n,-}^{\Delta x}-\mathbf{V}_{n,+}^{\Delta x}\right), \mathbf{U}_{\mathbf{V}}(\theta)\left(\mathbf{V}_{n,-}^{\Delta x}-\mathbf{V}_{n,+}^{\Delta x}\right)\right\rangle d \theta .
\end{aligned}
$$

Thus, we obtain

$$
\begin{aligned}
\mathcal{B}_{\mathrm{DG}}^{t}\left(\mathbf{V}^{\Delta x}, \mathbf{V}^{\Delta x}\right)= & \int_{\Omega} S\left(\mathbf{U}\left(\mathbf{V}^{\Delta x}\left(x, t_{-}^{N}\right)\right)\right) \mathrm{d} x-\int_{\Omega} S\left(\mathbf{U}\left(\mathbf{V}^{\Delta x}\left(x, t_{-}^{0}\right)\right)\right) \mathrm{d} x \\
& +\sum_{n, K} \int_{K} \int_{0}^{1} \theta\left\langle\left(\mathbf{V}_{n,-}^{\Delta x}-\mathbf{V}_{n,+}^{\Delta x}\right), \mathbf{U}_{\mathbf{V}}(\theta)\left(\mathbf{V}_{n,-}^{\Delta x}-\mathbf{V}_{n,+}^{\Delta x}\right)\right\rangle d \theta \mathrm{d} x \\
\geq & \int_{\Omega} S\left(\mathbf{U}^{\Delta}\left(\mathbf{V}^{\Delta x}\left(x, t_{-}^{N}\right)\right)\right) \mathrm{d} x-\int_{\Omega} S\left(\mathbf{U}\left(\mathbf{V}^{\Delta x}\left(x, t_{-}^{0}\right)\right)\right) \mathrm{d} x \\
& +\sum_{n, K} \frac{\lambda_{1}}{2} \int_{K}\left\|\mathbf{V}_{n,-}^{\Delta x}-\mathbf{V}_{n,+}^{\Delta x}\right\|^{2} \mathrm{~d} x \\
\geq & \int_{\Omega} S\left(\mathbf{U}\left(\mathbf{V}^{\Delta x}\left(x, t_{-}^{N}\right)\right)\right) \mathrm{d} x-\int_{\Omega} S\left(\mathbf{U}\left(\mathbf{V}^{\Delta x}\left(x, t_{-}^{0}\right)\right)\right) \mathrm{d} x
\end{aligned}
$$


The proof is finished by combining the four claims:

$$
\begin{aligned}
\mathcal{B}_{\mathrm{DG}}\left(\mathbf{V}^{\Delta x}, \mathbf{V}^{\Delta x}\right)+\mathcal{B}_{\mathrm{SD}}\left(\mathbf{V}^{\Delta x}, \mathbf{V}^{\Delta x}\right)+\mathcal{B}_{\mathrm{SC}}\left(\mathbf{V}^{\Delta x}, \mathbf{V}^{\Delta x}\right)=0 \\
\Rightarrow \mathcal{B}_{\mathrm{DG}}^{t}\left(\mathbf{V}^{\Delta x}, \mathbf{V}^{\Delta x}\right)+\mathcal{B}_{\mathrm{DG}}^{s}\left(\mathbf{V}^{\Delta x}, \mathbf{V}^{\Delta x}\right)+\mathcal{B}_{\mathrm{SD}}\left(\mathbf{V}^{\Delta x}, \mathbf{V}^{\Delta x}\right)+\mathcal{B}_{\mathrm{SC}}\left(\mathbf{V}^{\Delta x}, \mathbf{V}^{\Delta x}\right)=0 \\
\Rightarrow \int_{\Omega} S\left(\mathbf{U}\left(\mathbf{V}^{\Delta x}\left(x, t_{-}^{N}\right)\right)\right) \mathrm{d} x-\int_{\Omega} S\left(\mathbf{U}\left(\mathbf{V}^{\Delta x}\left(x, t_{-}^{0}\right)\right)\right) \mathrm{d} x \leq 0 \\
\Rightarrow \int_{\Omega} S\left(\mathbf{U}\left(\mathbf{V}^{\Delta x}\left(x, t_{-}^{N}\right)\right)\right) \mathrm{d} x \leq \int_{\Omega} S\left(\mathbf{U}\left(\mathbf{V}^{\Delta x}\left(x, t_{-}^{0}\right)\right)\right) \mathrm{d} x .
\end{aligned}
$$

Remark 3.2. Observe that Theorem 3.1 implies the stability of the total entropy only for the specific entropy pair chosen to design the entropy stable fluctuations.

\section{NumERICAL EXPERIMENTS}

In this section, the method (2.2) is applied to several test problems. We will approximate the two-layer shallow water equations as a prototypical example of non-conservative hyperbolic systems. We start with onedimensional problems and, for simplicity, the notational reference to the dimension is suppressed, e.g. we will write $A$ instead of $A^{1}$. The parameters in the scheme are set to $C^{\mathrm{SD}}=10, C^{\mathrm{SC}}=1$, and $\bar{C}^{\mathrm{SC}}=0$ and a CFL number of 0.5 is applied. The shock-capturing streamline diffusion space-time DG method leads to a system of nonlinear algebraic equations at each time step. These equations are solved with a damped Newton method as described in $[17,18]$.

\subsection{Two-layer shallow water equations}

As a first example, we consider the two-layer shallow water equations that model the flow of two superposed immiscible fluid layers (see [9]):

$$
\begin{aligned}
\left(h_{1}\right)_{t}+\left(h_{1} u_{1}\right)_{x} & =0 \\
\left(h_{2}\right)_{t}+\left(h_{2} u_{2}\right)_{x} & =0 \\
\left(h_{1} u_{1}\right)_{t}+\left(\frac{1}{2} g h_{1}^{2}+h_{1} u_{1}^{2}\right)_{x} & =-g h_{1}\left(h_{2}\right)_{x} \\
\left(h_{2} u_{2}\right)_{t}+\left(\frac{1}{2} g h_{2}^{2}+h_{2} u_{2}^{2}\right)_{x} & =-g h_{2}\left(r h_{1}\right)_{x} .
\end{aligned}
$$

Here, $h_{1}$ and $h_{2}$ represent the thickness of the layers, while $u_{1}$ and $u_{2}$ represent their depth-averaged velocities, $g$ is the gravitational constant, and $r$ is the ratio of the layer densities $\rho_{1}$ and $\rho_{2}$.

In order to write (4.1) in the nonconservative form (1.1), we define

$$
\mathbf{U}=\left(\begin{array}{c}
h_{1} \\
h_{2} \\
h_{1} u_{1} \\
h_{2} u_{2}
\end{array}\right), \quad A(\mathbf{U})=\left(\begin{array}{cccc}
0 & 0 & 1 & 0 \\
0 & 0 & 0 & 1 \\
g h_{1}-u_{1}^{2} & g h_{1} & 2 u_{1} & 0 \\
r g h_{2} & g h_{2}-u_{2}^{2} & 0 & 2 u_{2}
\end{array}\right)
$$

An entropy pair for the two-layer shallow water equations is given by

$$
S(\mathbf{U})=\sum_{j=1}^{2} \rho_{j}\left(\frac{1}{2} h_{j} u_{j}^{2}+\frac{1}{2} g h_{j}^{2}\right)+g \rho_{1} h_{1} h_{2},
$$




$$
Q(\mathbf{U})=\sum_{j=1}^{2} \rho_{j}\left(\frac{1}{2} h_{j} u_{j}^{2}+g h_{j}^{2}\right) u_{j}+g \rho_{1} h_{1} h_{2}\left(u_{1}+u_{2}\right) .
$$

Thus, we reformulate (4.1) for the computation in terms of the entropy variables

$$
\mathbf{V}=\left(\begin{array}{c}
\rho_{1}\left(-\frac{1}{2} u_{1}^{2}+g\left(h_{1}+h_{2}\right)\right) \\
\rho_{2}\left(-\frac{1}{2} u_{2}^{2}+g h_{2}\right)+\rho_{1} g h_{1} \\
\rho_{1} u_{1} \\
\rho_{2} u_{2}
\end{array}\right)
$$

We use fluctuations that are based on the scheme presented in [9]. They are given by

$$
\mathbf{D}^{-}\left(\mathbf{V}_{-}, \mathbf{V}_{+} ; \nu\right)=\left(\mathbb{F}^{*}\left(\mathbf{V}_{-}, \mathbf{V}_{+}\right)-\mathbf{F}\left(\mathbf{V}_{-}\right)+\mathbf{B}\left(\mathbf{V}_{-}, \mathbf{V}_{+}\right)\right) \nu
$$

where $\nu= \pm 1$ and

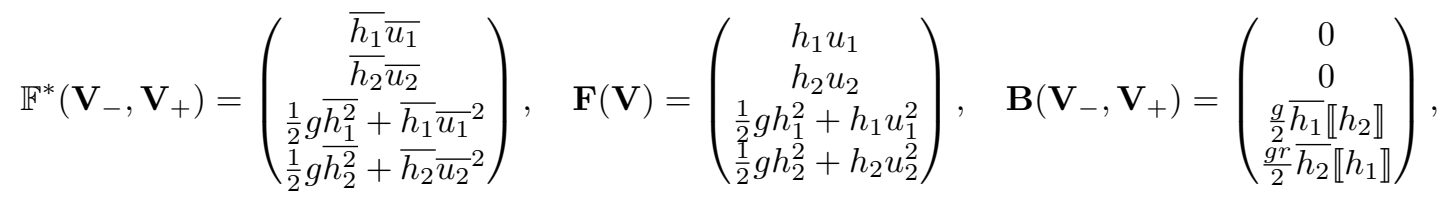

where

$$
\bar{w}=\frac{w_{-}+w_{+}}{2}, \quad \llbracket w \rrbracket=w_{+}-w_{-},
$$

for any variable $w$. These fluctuations satisfy the consistency conditions (2.4) and (2.5) for the family of straight segments (1.11), as well as the entropy conservative condition (2.7). Moreover, the Rusanov-type diffusion (2.11) is added to the fluctuations.

\subsubsection{Smooth solution}

We start with a smooth solution to test the high-order accuracy of the schemes. For this problem, we set $g=1 / 2$ and $r=0.9$. The initial data is

$$
\begin{array}{ll}
h_{1}=1+0.5 \exp \left(-(5 x)^{2}\right), & u_{1}=0, \\
h_{2}=1+0.5 \exp \left(-(5 x)^{2}\right), & u_{2}=0,
\end{array}
$$

and we compute on the domain $[-1,1]$ up to the time $T=0.2$ so that the solution remains smooth. The reference solution was computed on a grid with 320000 cells using centered finite differences and SSP RK2.

Figure 1 depicts the convergence for various polynomial degrees $p$ for scheme (2.2). A convergence rate of almost $p+1$ is observed, demonstrating the high order of accuracy of the scheme. Moreover, even on the coarsest meshes, the amplitude of the error is much smaller for higher polynomial degrees, clearly indicating the advantage of using high order methods over lower order methods, at least in this smooth test case.

\subsubsection{Riemann problem}

Next, we investigate the numerical approximation of weak solutions of the two-layer shallow water equations. As discussed in the introduction, the definition of weak solutions depends explicitly on the choice of the family 


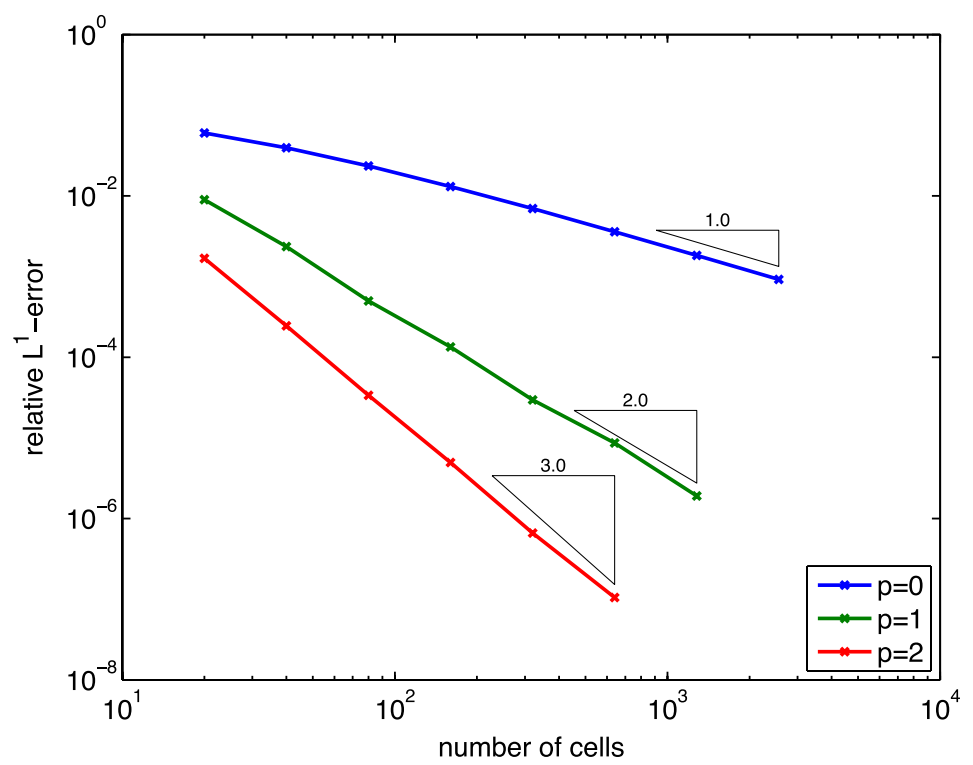

Figure 1. Order of convergence of the scheme for the two-layer shallow water model with a smooth solution.

of paths that connects the left and right states across a discontinuity. A particularly good choice of path will correspond to a (reparametrization of) the viscous profile for the underlying parabolic system.

Following [9] and references therein, we consider (4.1) as the vanishing-viscosity limit of the system:

$$
\begin{aligned}
\left(h_{1}\right)_{t}+\left(h_{1} u_{1}\right)_{x} & =0 \\
\left(h_{2}\right)_{t}+\left(h_{2} u_{2}\right)_{x} & =0 \\
\left(h_{1} u_{1}\right)_{t}+\left(\frac{1}{2} g h_{1}^{2}+h_{1} u_{1}^{2}\right)_{x} & =-g h_{1}\left(h_{2}\right)_{x}+\epsilon\left(h_{1}\left(u_{1}\right)_{x}\right)_{x} \\
\left(h_{2} u_{2}\right)_{t}+\left(\frac{1}{2} g h_{2}^{2}+h_{2} u_{2}^{2}\right)_{x} & =-g h_{2}\left(r h_{1}\right)_{x}+\epsilon\left(h_{2}\left(u_{2}\right)_{x}\right)_{x} .
\end{aligned}
$$

To write (4.8) in the viscous form (1.10) we must define

$$
R(\mathbf{U})=\left(\begin{array}{cccc}
0 & 0 & 0 & 0 \\
0 & 0 & 0 & 0 \\
-u_{1} & 0 & 1 & 0 \\
0 & -u_{2} & 0 & 1
\end{array}\right)
$$

Let us consider the Riemann problem with initial data given by

$$
\mathbf{U}_{l}=\left(\begin{array}{c}
1.376 \\
0.6035 \\
0.04019 \\
-0.04906
\end{array}\right), \quad \mathbf{U}_{r}=\left(\begin{array}{c}
0.37 \\
1.593 \\
-0.1868 \\
0.1742
\end{array}\right)
$$

As in [9], we consider $g=9.81$ and $r=0.98$. The spatial domain is the interval $[0,1]$ with the initial discontinuity located at $x=0.5$. The flow is evolved up to the time $T=1$. The reference solution was computed by discretising the equation for the viscous profiles corresponding to the regularization in (4.8): see Appendix A for details. 
Figure 2 shows the numerical approximation of $h_{2}$ (that corresponds to the interface between the two layers) for different values of $p$ and number of cells. These approximations are compared against the reference solution. Notice that, for $p=0$ (piecewise constant basis functions), the Rusanov diffusion operator leads to very smeared profiles. This is due to the fact that, for the chosen values of the constants, the system possess two different pair of eigenvalues: the so-called external ones that correspond to barotropic waves, and the internal ones, that correspond to baroclinic waves. The external eigenvalues are much larger in amplitude than the internal ones, thus the slower baroclinic waves are more smeared by the numerical viscosity. The waves shown in Figure 2 are internal: the external waves have exited the domain before $t=1$. We observe that, for $p=1$ and $p=2$, the intermediate state is approximated quite well, albeit with some oscillations in the case $p=2$. In the zooms shown in figures (d), (e), and (f) the convergence error can be clearly observed in the intermediate state. However, this error decreases with the order of the numerical method.

We remark that the oscillations appearing in Figure 2 are small (they are hardly visible on the non-zoomed image). Similar oscillations appear in the case of systems of conservation laws (see [18]) although they are of slightly lower amplitude in that case.

\subsection{Two-layer shallow water equations in $2 \mathrm{D}$}

The two-layer shallow-water equations in two space dimensions are

$$
\begin{aligned}
\left(h_{1}\right)_{t}+\left(h_{1} u_{1}\right)_{x}+\left(h_{1} v_{1}\right)_{y} & =0, \\
\left(h_{2}\right)_{t}+\left(h_{2} u_{2}\right)_{x}+\left(h_{2} v_{2}\right)_{y} & =0, \\
\left(h_{1} u_{1}\right)_{t}+\left(\frac{1}{2} g h_{1}^{2}+h_{1} u_{1}^{2}\right)_{x}+\left(h_{1} u_{1} v_{1}\right)_{y} & =-g h_{1}\left(h_{2}\right)_{x}, \\
\left(h_{2} u_{2}\right)_{t}+\left(\frac{1}{2} g h_{2}^{2}+h_{2} u_{2}^{2}\right)_{x}+\left(h_{2} u_{2} v_{2}\right)_{y} & =-g h_{2}\left(r h_{1}\right)_{x}, \\
\left(h_{1} v_{1}\right)_{t}+\left(h_{1} u_{1} v_{1}\right)_{x}+\left(\frac{1}{2} g h_{1}^{2}+h_{1} v_{1}^{2}\right)_{y} & =-g h_{1}\left(h_{2}\right)_{y}, \\
\left(h_{2} v_{2}\right)_{t}+\left(h_{2} u_{2} v_{2}\right)_{x}+\left(\frac{1}{2} g h_{2}^{2}+h_{2} v_{2}^{2}\right)_{y} & =-g h_{2}\left(r h_{1}\right)_{y} .
\end{aligned}
$$

Here again, $h_{1}$ and $h_{2}$ represent the thicknesses of the upper and lower layer, respectively; $u_{i}$ and $v_{i}$ are the velocities of the $i$ th layer in $x$ and $y$ direction, respectively; $g$ is the gravitational constant; and $r=\rho_{1} / \rho_{2}$ the density ratio of the layers.

In order to write (4.11) in the nonconservative form (1.1), we define

$$
\begin{aligned}
& \mathbf{U}=\left(h_{1}, h_{2}, h_{1} u_{1}, h_{2} u_{2}, h_{1} v_{1}, h_{2} v_{2}\right)^{\mathrm{T}}, \\
& A^{1}(\mathbf{U})=\left(\begin{array}{cccccc}
0 & 0 & 1 & 0 & 0 & 0 \\
0 & 0 & 0 & 1 & 0 & 0 \\
g h_{1}-u_{1}^{2} & g h_{1} & 2 u_{1} & 0 & 0 & 0 \\
r g h_{2} & g h_{2}-u_{2}^{2} & 0 & 2 u_{2} & 0 & 0 \\
-u_{1} u_{2} & 0 & v_{1} & 0 & u_{1} & 0 \\
0 & -u_{2} v_{2} & 0 & v_{2} & 0 & u_{2}
\end{array}\right), A^{2}(\mathbf{U})=\left(\begin{array}{cccccc}
0 & 0 & 0 & 0 & 1 & 0 \\
0 & 0 & 0 & 0 & 0 & 1 \\
-u_{1} u_{2} & 0 & v_{1} & 0 & u_{1} & 0 \\
0 & -u_{2} v_{2} & 0 & v_{2} & 0 & u_{2} \\
g h_{1}-v_{1}^{2} & g h_{1} & 0 & 0 & 2 v_{1} & 0 \\
r g h_{2} & g h_{2}-v_{2}^{2} & 0 & 0 & 0 & 2 v_{2}
\end{array}\right) .
\end{aligned}
$$

An entropy pair for the two-layer shallow water equations is given by

$$
\begin{aligned}
S(\mathbf{U}) & =\sum_{j=1}^{2} \rho_{j}\left(\frac{1}{2} h_{j} u_{j}^{2}+\frac{1}{2} h_{j} v_{j}^{2}+\frac{1}{2} g h_{j}^{2}\right)+g \rho_{1} h_{1} h_{2}, \\
Q^{1}(\mathbf{U}) & =\sum_{j=1}^{2} \rho_{j}\left(\frac{1}{2} h_{j} u_{j}^{2}+\frac{1}{2} h_{j} v_{j}^{2}+g h_{j}^{2}\right) u_{j}+g \rho_{1} h_{1} h_{2}\left(u_{1}+u_{2}\right), \\
Q^{2}(\mathbf{U}) & =\sum_{j=1}^{2} \rho_{j}\left(\frac{1}{2} h_{j} u_{j}^{2}+\frac{1}{2} h_{j} v_{j}^{2}+g h_{j}^{2}\right) v_{j}+g \rho_{1} h_{1} h_{2}\left(v_{1}+v_{2}\right) .
\end{aligned}
$$




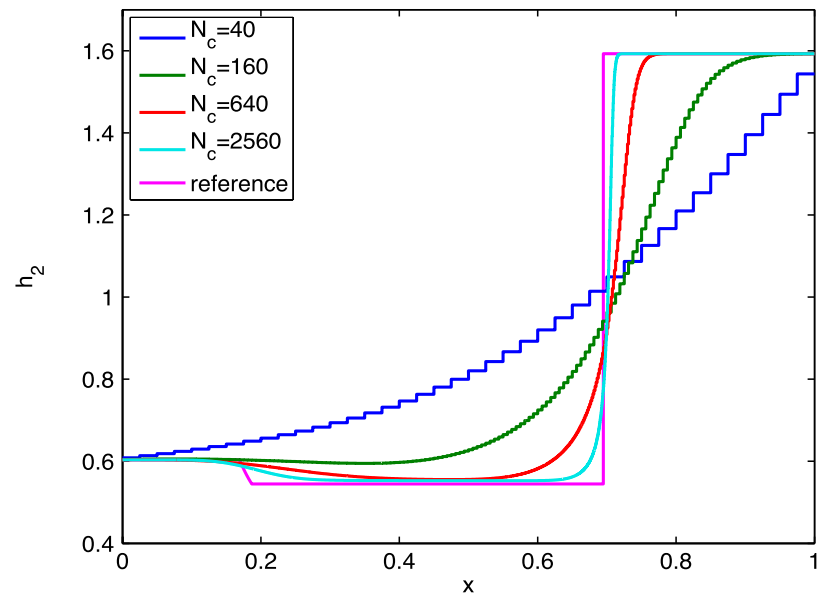

(a) $h_{2}, p=0$

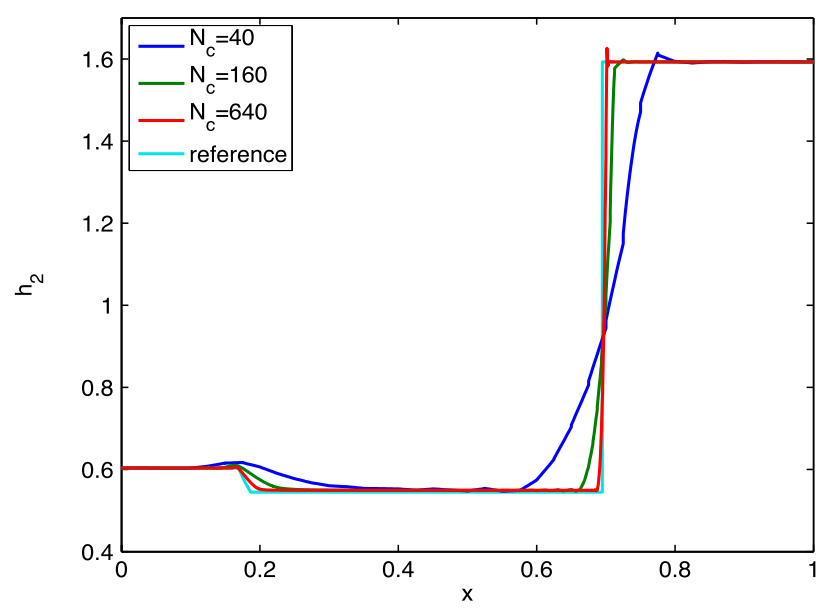

(c) $h_{2}, p=1$

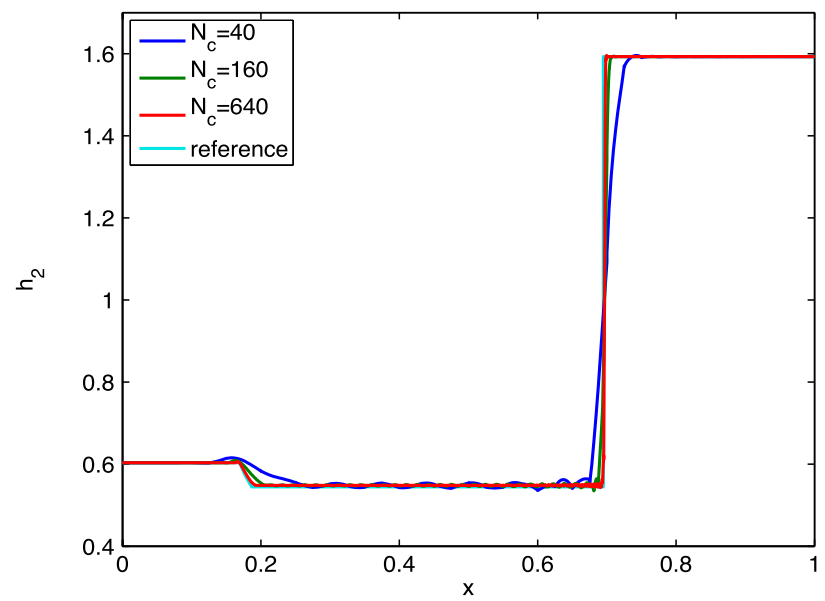

(e) $h_{2}, p=2$

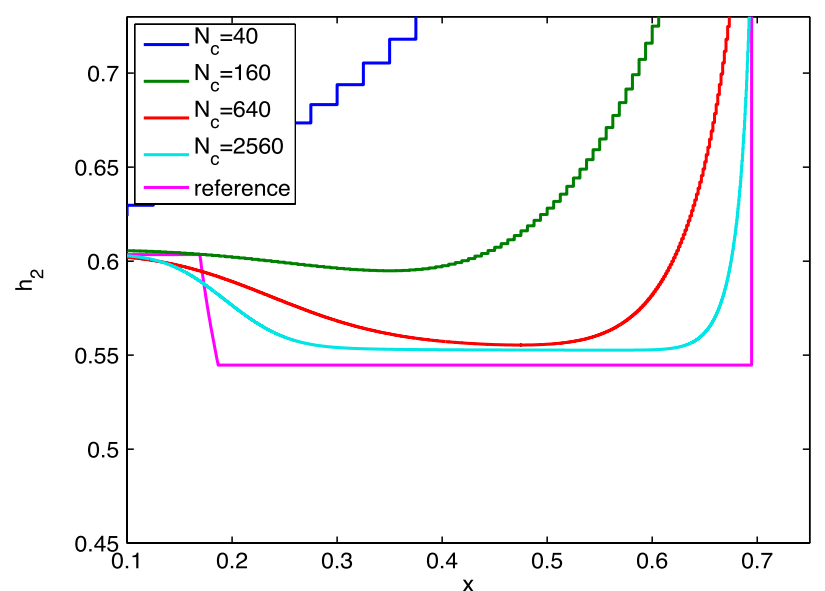

(b) $h_{2}, p=0$ (close up)

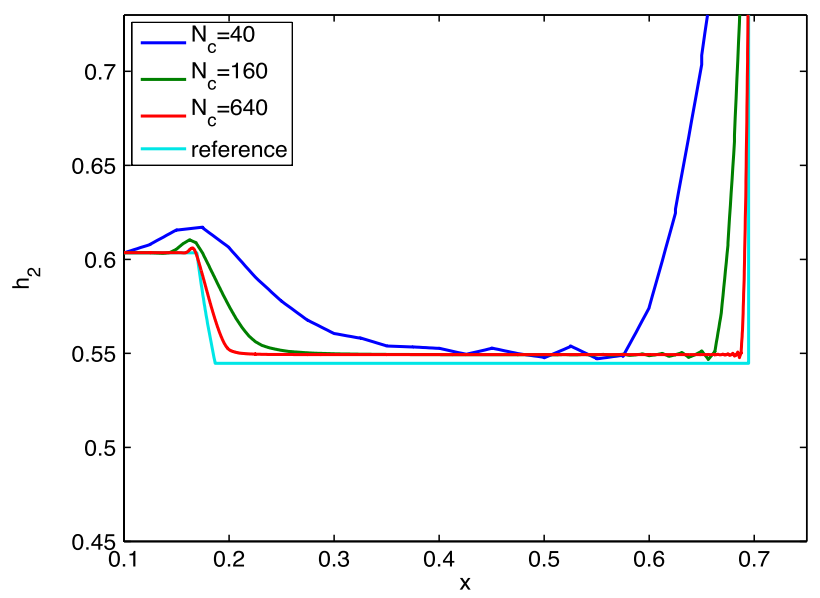

(d) $h_{2}, p=1$ (close up)

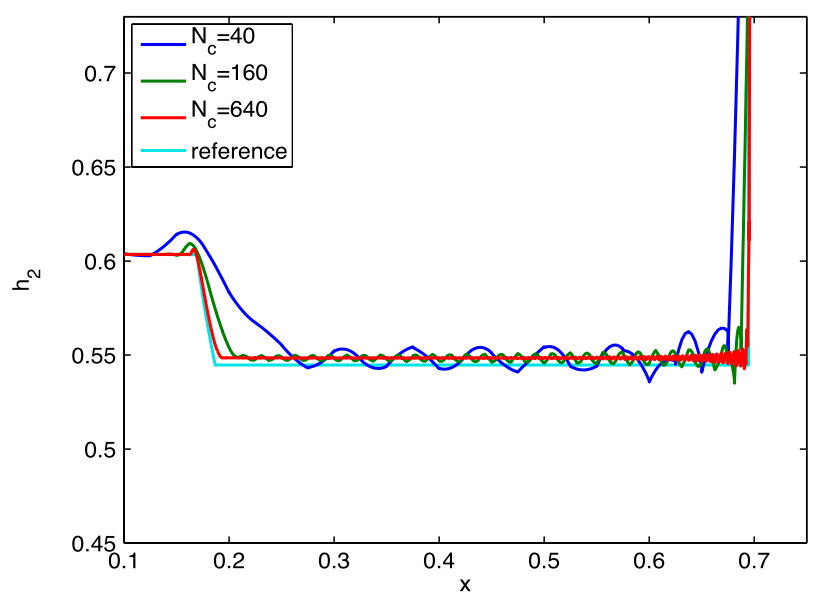

(f) $h_{2}, p=2$ (close up)

Figure 2. Approximation of $h_{2}$ for the two-layer shallow water equation with initial conditions (4.10) at time $t=1$ obtained with the $\mathrm{DG}+\mathrm{SD}+\mathrm{SC}$ scheme using different degrees of polynomial $p$ and number of cells $N_{c}$. 
Thus, system (4.11) is written in terms of the entropy variables

$$
\mathbf{V}=\left(\rho_{1}\left(-\frac{1}{2} u_{1}^{2}-\frac{1}{2} v_{1}^{2}+g\left(h_{1}+h_{2}\right)\right), \rho_{2}\left(-\frac{1}{2} u_{2}^{2}-\frac{1}{2} v_{2}^{2}+g h_{2}\right)+\rho_{1} g h_{1}, \rho_{1} u_{1}, \rho_{2} u_{2}, \rho_{1} v_{1}, \rho_{2} v_{2}\right)^{\mathrm{T}} .
$$

The entropy-conservative fluctuations are given now by

$$
\mathbf{D}^{-}\left(\mathbf{V}_{-}, \mathbf{V}_{+} ; \nu\right)=\sum_{k=1}^{2}\left(\mathbb{F}^{k, *}\left(\mathbf{V}_{-}, \mathbf{V}_{+}\right)-\mathbf{F}^{k}\left(\mathbf{V}_{-}\right)+\mathbf{B}^{k}\left(\mathbf{V}_{-}, \mathbf{V}_{+}\right)\right) \nu^{k}
$$

where

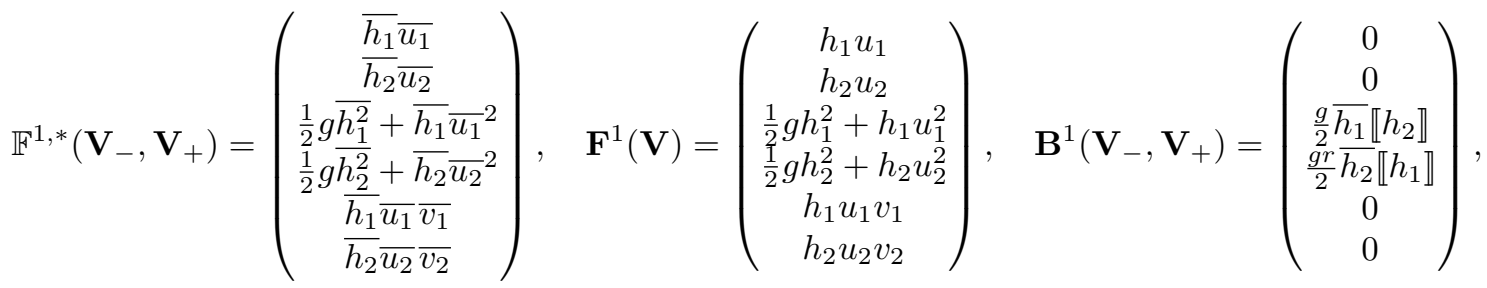

$$
\begin{aligned}
& \mathbb{F}^{2, *}\left(\mathbf{V}_{-}, \mathbf{V}_{+}\right)=\left(\begin{array}{c}
\overline{h_{1}} \overline{v_{1}} \\
\overline{h_{2}} \overline{v_{2}} \\
\overline{h_{1}} \overline{u_{1}} \overline{v_{1}} \\
\overline{h_{2}} \overline{u_{2}} \overline{v_{2}} \\
\frac{1}{2} g \overline{h_{1}^{2}}+\overline{h_{1}} \bar{v}_{1}^{2} \\
\frac{1}{2} g \overline{h_{2}^{2}}+\overline{h_{2}}{\overline{v_{2}}}^{2}
\end{array}\right), \quad \mathbf{F}^{2}(\mathbf{V})=\left(\begin{array}{c}
h_{1} v_{1} \\
h_{2} v_{2} \\
h_{1} u_{1} v_{1} \\
h_{2} u_{2} v_{2} \\
\frac{1}{2} g h_{1}^{2}+h_{1} v_{1}^{2} \\
\frac{1}{2} g h_{2}^{2}+h_{2} v_{2}^{2}
\end{array}\right), \quad \mathbf{B}^{2}\left(\mathbf{V}_{-}, \mathbf{V}_{+}\right)=\left(\begin{array}{c}
0 \\
0 \\
0 \\
0 \\
\frac{g}{2} \overline{h_{1}} \llbracket h_{2} \rrbracket \\
\frac{g r}{2} \overline{h_{2}} \llbracket h_{1} \rrbracket
\end{array}\right) .
\end{aligned}
$$

\subsection{Vortex advection}

We consider the following smooth solution introduced in [13] to study the convergence properties:

$$
\begin{aligned}
& h_{1}=\frac{v_{10}^{2} s_{2} \exp \left(2 s_{1}\right)\left(1-\exp \left(-2 s_{1} r^{2}\right)\right)-v_{20}^{2} s_{1} \exp \left(2 s_{2}\right)\left(1-\exp \left(-2 s_{2} r^{2}\right)\right)+4 h_{10} g s_{1} s_{2}(1-\rho)}{4 g(1-\rho) s_{1} s_{2}}, \\
& h_{2}=\frac{v_{20}^{2} s_{1} \exp \left(2 s_{2}\right)\left(1-\exp \left(-2 s_{2} r^{2}\right)\right)-\rho v_{10}^{2} s_{2} \exp \left(2 s_{1}\right)\left(1-\exp \left(-2 s_{1} r^{2}\right)\right)+4 h_{20} g s_{1} s_{2}(1-\rho)}{4 g(1-\rho) s_{1} s_{2}}, \\
& u_{1}=\bar{u}-v_{1}^{\theta} \sin \theta, \quad v_{1}=\bar{v}+v_{1}^{\theta} \cos \theta, \\
& u_{2}=\bar{u}-v_{2}^{\theta} \sin \theta, \quad v_{2}=\bar{v}+v_{2}^{\theta} \cos \theta,
\end{aligned}
$$

where

$$
\begin{aligned}
& v_{1}^{\theta}=r v_{10} \exp \left(s_{1}\left(1-r^{2}\right)\right), \quad v_{2}^{\theta}=r v_{20} \exp \left(s_{2}\left(1-r^{2}\right)\right) \\
& r=\sqrt{\left(x-\bar{u} t-x_{c}\right)^{2}+\left(y-\bar{v} t-y_{c}\right)^{2}} \\
& \cos \theta=\left(x-\bar{u} t-x_{c}\right) / r, \quad \sin \theta=\left(y-\bar{v} t-y_{c}\right) / r
\end{aligned}
$$

The parameters are given by:

$$
\begin{aligned}
& x_{c}=5, \quad y_{c}=5, \quad \bar{u}=5, \quad \bar{v}=5, \quad s_{1}=\frac{1}{2}, \quad s_{2}=1, \\
& v_{10}=\frac{3}{4}, \quad v_{20}=\frac{1}{10}, \quad h_{10}=1, \quad h_{20}=1, \quad g=10, \quad \rho=\frac{\rho_{1}}{\rho_{2}}=0.9 .
\end{aligned}
$$



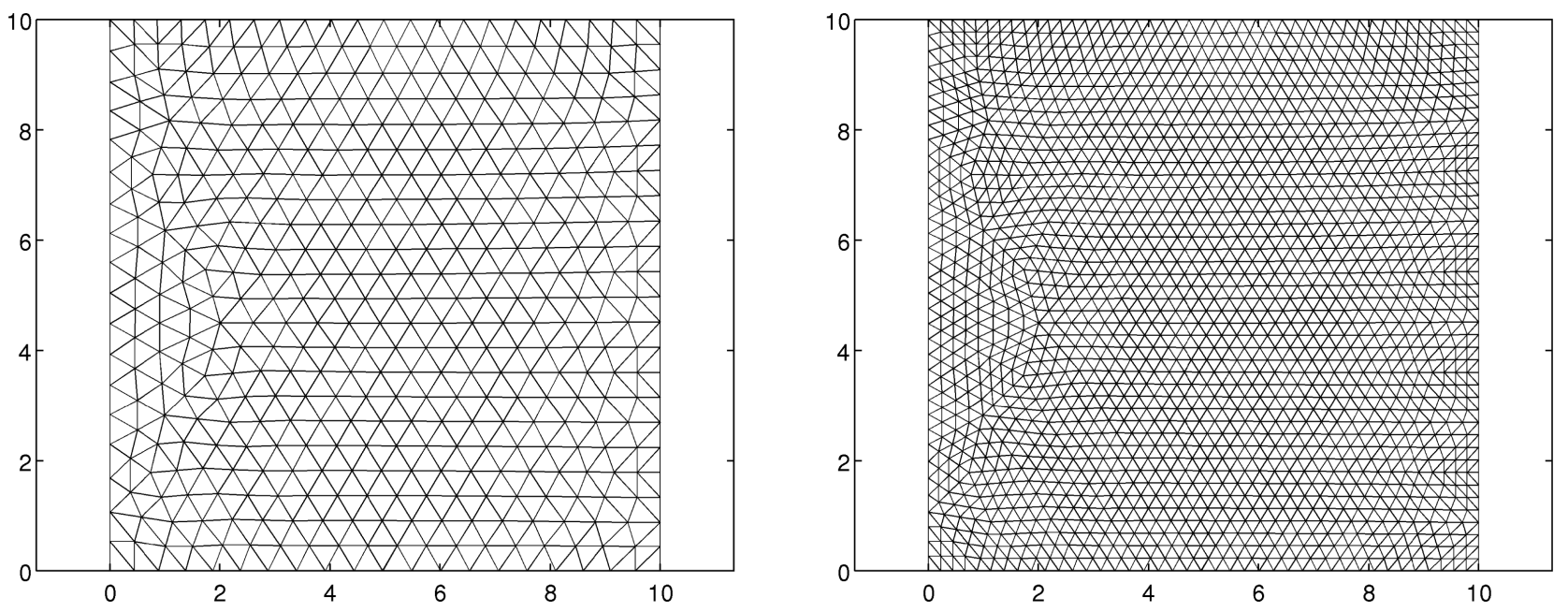

FiguRE 3. Unstructured mesh used for the vortex advection problem. Left: 828 spatial cells and right: 3312 spatial cells.

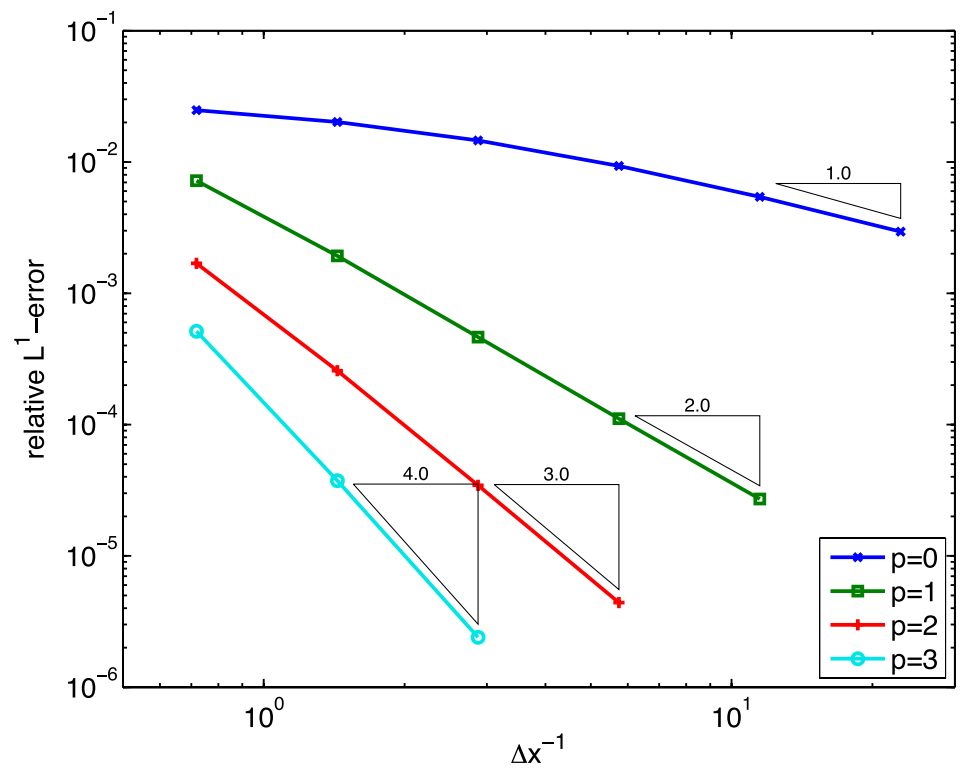

FiguRE 4. Convergence of the scheme for the vortex advection problem.

The solutions represents the advection of a vortex, whose initial position is given by $\left(x_{c}, y_{c}\right)$, with velocity $(\bar{u}, \bar{v})$. $h_{10}$ and $h_{20}$ are the heights of the layer at the vortex, and $v_{10}$ and $v_{20}$ is the angular velocity around the vortex. We consider the domain $[0,10]^{2}$ and compute up to the time $T=0.5$.

We run the $\mathrm{DG}+\mathrm{SD}+\mathrm{SC}$ scheme on a sequence of unstructured grids of the form shown in Figure 3. Figure 4 shows the convergence for various polynomial degrees $p$ for scheme (2.2). We can observe that the schemes achieve the order of accuracy of $p+1$, even on these unstructured grids. In addition, a higher polynomial degree $p$ leads also to a lower amplitude of error, even on coarse meshes. 


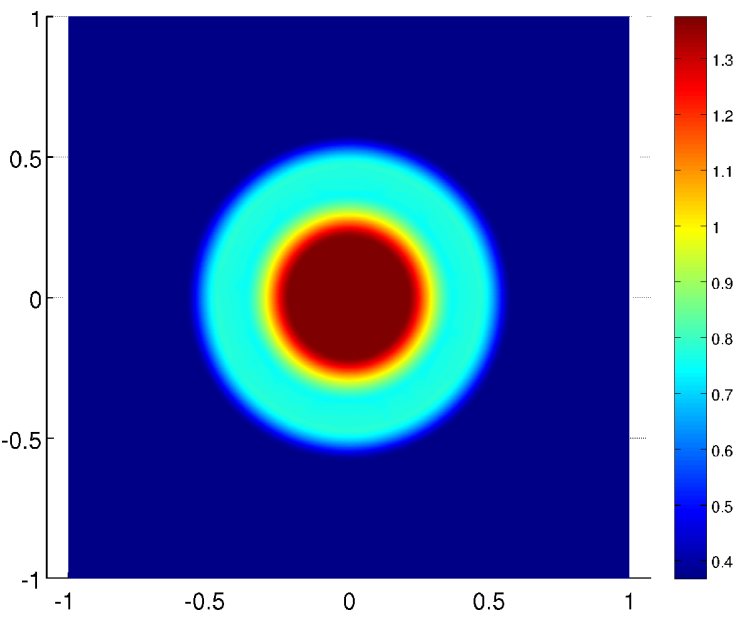

(a) $h_{1}$

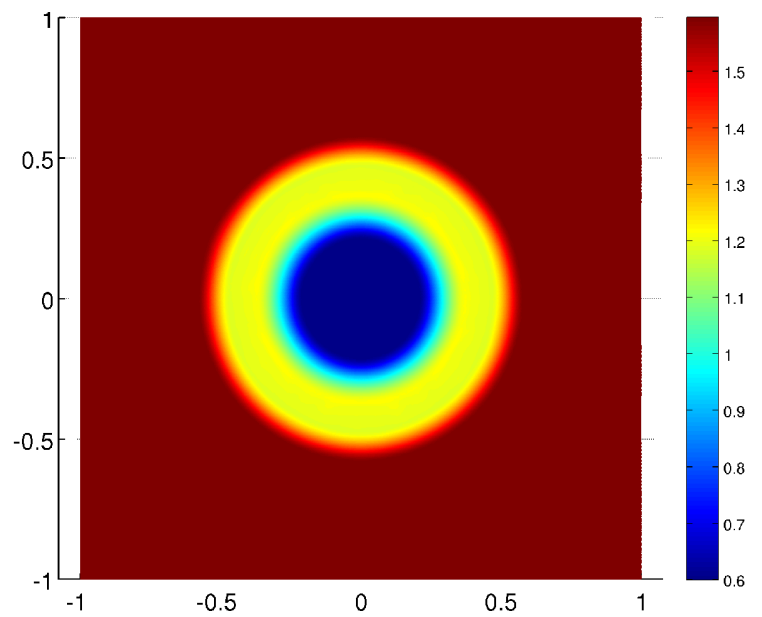

(b) $h_{2}$

FiguRE 5. Approximate solutions for the radial dam break problem with $p=1$ and $N_{c}=53760$.

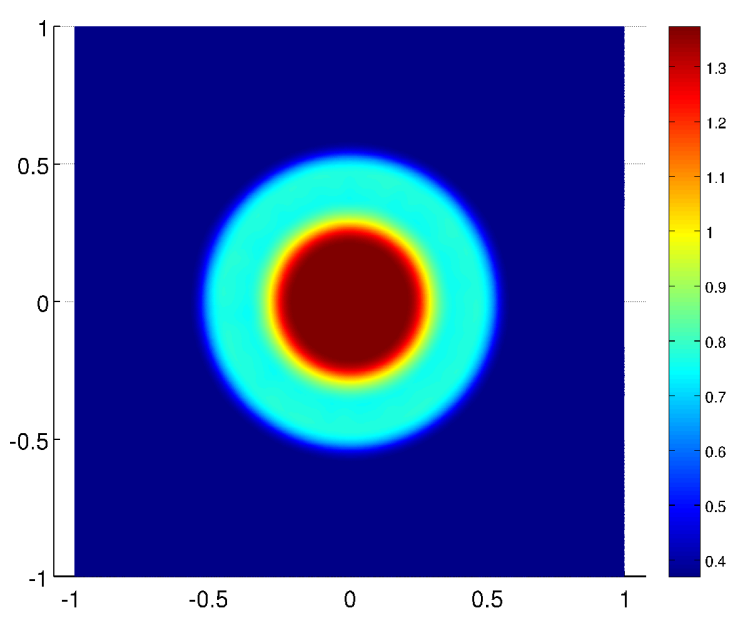

(a) $h_{1}$

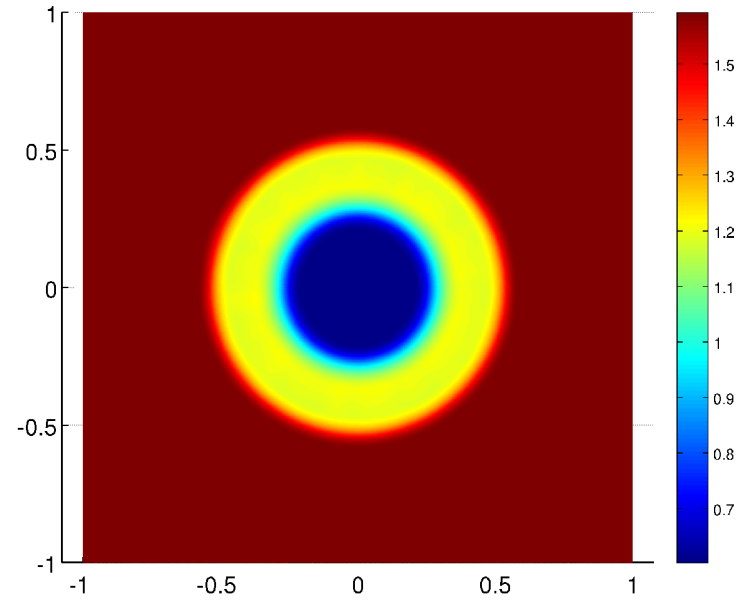

(b) $h_{2}$

Figure 6. Approximate solutions for the radial dam break problem with $p=2$ and $N_{c}=13440$.

\subsection{Radial dam break problem}

We consider the following radial dam break problem, which leads only to small fast external waves, but large amplitude yet slow moving internal waves. The domain is $[-1,1]^{2}$. The initial data is as follows:

$$
h_{1}=\left\{\begin{array}{ll}
1.376, & r \leq r_{0}, \\
0.37, & r>r_{0},
\end{array} \quad h_{2}= \begin{cases}0.6035, & r \leq r_{0}, \\
1.593, & r>r_{0},\end{cases}\right.
$$

where $r=\sqrt{x^{2}+y^{2}}$ and $r_{0}=0.4$. All the initial velocities are zero: $u_{1}=u_{2}=v_{1}=v_{2}=0$. We compute up to the time $T=0.4$, by which the external waves have left the domain. 


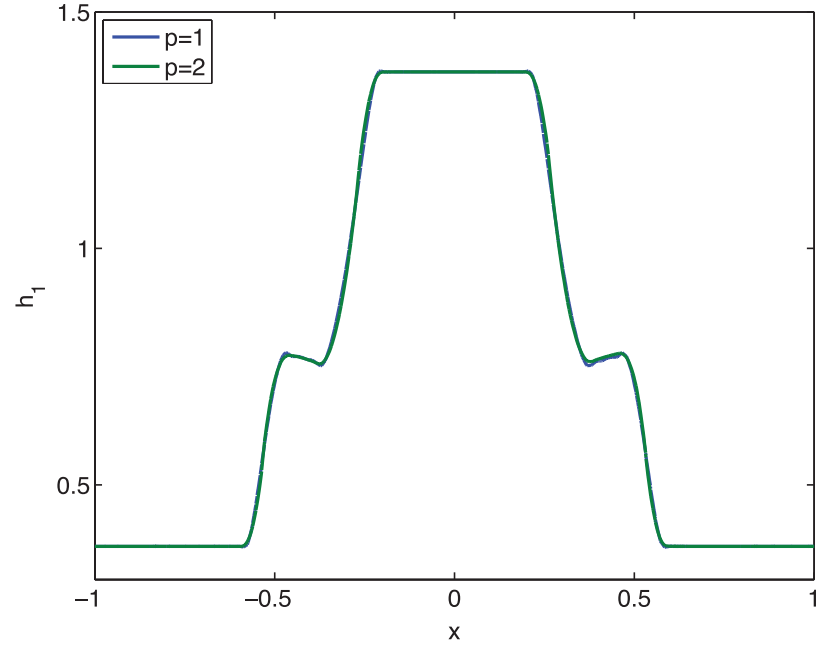

(a) $h_{1}$

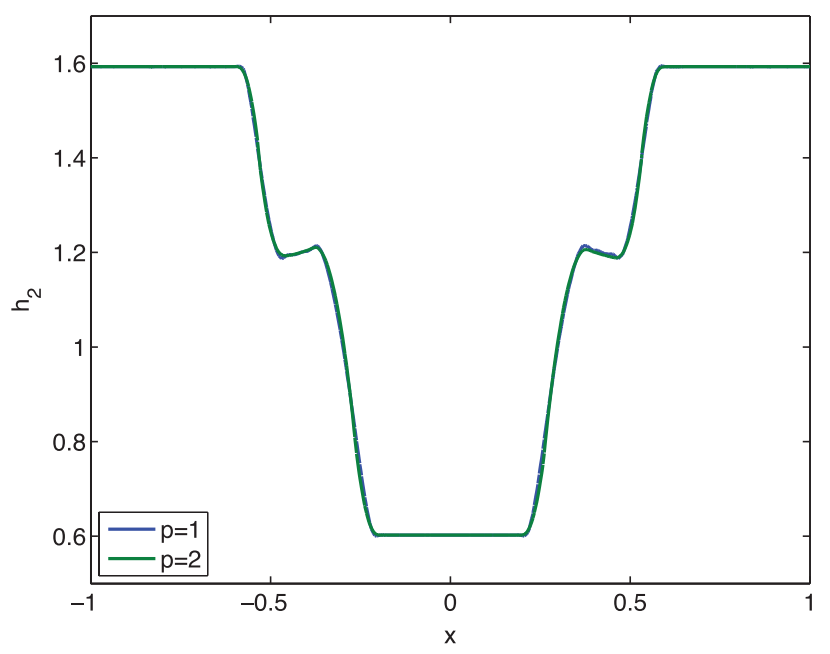

(b) $h_{2}$

FiguRE 7 . Slices in $x$ direction of the approximate solutions for the radial dam break problem with $p=1$ and $N_{c}=53760$ as well as $p=2$ and $N_{c}=13440$.

Figures 5 and 6 show the approximate heights of both layers for $p=1$ and $p=2$, respectively, obtained with the $\mathrm{DG}+\mathrm{SD}+\mathrm{SC}$ scheme using an unstructured triangular mesh, similar in form to the grids shown in Figure 3. In addition, Figure 7 shows slices of these solutions in the $x$ direction. We can observe that the structure of the solution is nicely approximated even though the discontinuities are slightly smeared as the meshes are not very fine. In particular, the solutions are radially symmetric, i.e. no distortion due to the unstructured mesh is visible. Furthermore, we can see the advantage of a higher polynomial degree as the results are very comparable even though only 13440 cells are used for $p=2$, where as the $p=1$ results are based on a finer grid with 53760 cells.

\section{Conclusion}

This paper deals with the extension to multidimensional nonconservative hyperbolic systems (1.1) of the space-time discontinuous Galerkin streamline diffusion shock capturing method introduced in [17, 18]. In this method, the equation was formulated in entropy variables and an entropy stable numerical flux was used to deal with the discontinuities of the test functions at the boundaries of the elements: those are the main ingredients to prove that a discrete entropy inequality is satisfied. In order to adapt the method to nonconservative systems, the path-conservative entropy-stable fluctuations introduced in [9] are used to replace the role played by the entropy-stable numerical flux. The discrete entropy inequality stands for the resulting method. Moreover, the method has order $p+1$ of accuracy for smooth solutions, where $p$ is the polynomial degree of the test functions in the elements, as it has been shown in the numerical experiments.

The accurate approximation of the physically correct solutions of a nonconservative systems is a challenging problem. This is due to the fact that their weak solutions depend explicitly on the underlying small-scale mechanisms like diffusion or dispersion. This dependency affects even to the very definition of weak solution, which is not unique: in order to fix the concept of weak solution the small-scale effects have to be introduced, either by means of the choice of a family of paths, as it is done in the theory of [12] or, equivalently, by considering the hyperbolic system as the vanishing-viscosity limit of a family of regularized problems. In this latter case, the correct family of paths is given by the viscous profiles of the regularized problems. 
Once the notion of weak solution has been set, the design of methods verifying that the limits of the numerical solutions are weak solutions according to the prescribed definition is difficult, due to the small-scale effects implicitly introduced at the numerical level which may be in disagreement with the physical ones. Due to this, numerical methods in which this small-scale effects are not controlled may fail in converging to the correct weak solutions, even if a formal consistency with their definition is imposed: see [7].

In [9] it has been shown that entropy stable numerical methods reduce in a significant way this lack of convergence phenomenon, even if they are not consistent with the correct family of paths but with an approximated one (as the straight-segments family) provided that the numerical viscosity is in good agreement with the viscous terms of the regularized equations. Therefore, the numerical method presented here, which can be considered as a high-order generalization of entropy-stable path-conservative methods were expected to inherit this property and, moreover, to reduce the convergence error with the increasing accuracy. The numerical experiments shown here confirm expectations.

Last but not least, we have proposed and checked an ansatz for computing reference solutions for Riemann problems related to 1D nonconservative systems by numerically approaching the viscous profiles of the regularized systems. This ansatz has been applied to the two-layer shallow-water system.

\section{Appendix A. Computation of Riemann solutions for NON-CONSERVATIVE SYSTEMS}

We describe here the ansatz followed to find reference solutions for Riemann problems related to the onedimensional two-layer shallow water system. This ansatz can be applied to any nonconservative hyperbolic system:

$$
\mathbf{U}_{t}+A(\mathbf{U}) \mathbf{U}_{x}=0
$$

with initial conditions

$$
\mathbf{U}(x, 0)= \begin{cases}\mathbf{U}_{l}, & x<0, \\ \mathbf{U}_{r}, & x \geq 0 .\end{cases}
$$

The system is supposed to be strictly hyperbolic and the characteristic fields are assumed to be either genuinely nonlinear or linearly degenerate, so that the solution of the Riemann problems consists of $N$ simple waves (shocks, rarefactions, or contact discontinuities).

To fix the ideas, let us consider the case $N=2$, i.e. $\mathbf{U} \in \mathbb{R}^{2}$, and a Riemann problem whose solution is composed by two shocks:

$$
\mathbf{U}(x, t)= \begin{cases}\mathbf{U}_{l}, & x<\sigma_{1} t \\ \mathbf{U}^{*}, & \sigma_{1} t \leq x<\sigma_{2} t \\ \mathbf{U}_{r}, & \sigma_{2} t \leq x\end{cases}
$$

where the shock speeds $\sigma_{1}$ and $\sigma_{2}$ as well as the intermediate state $\mathbf{U}^{*}$ are unknown. If the system was conservative, the following $4 \times 4$ nonlinear system would have to be solved to find these unknowns:

$$
\begin{aligned}
& \sigma_{1}\left(\mathbf{U}^{*}-\mathbf{U}_{l}\right)=\mathbf{F}\left(\mathbf{U}^{*}\right)-\mathbf{F}\left(\mathbf{U}_{l}\right), \\
& \sigma_{2}\left(\mathbf{U}_{r}-\mathbf{U}^{*}\right)=\mathbf{F}\left(\mathbf{U}_{r}\right)-\mathbf{F}\left(\mathbf{U}^{*}\right) .
\end{aligned}
$$


Now for the non-conservative case, (A.4) needs to be replaced by the generalized Rankine-Hugoniot conditions

$$
\begin{aligned}
\sigma_{1}\left(\mathbf{U}^{*}-\mathbf{U}_{l}\right) & =\int_{0}^{1} A\left(\Phi\left(s ; \mathbf{U}_{l}, \mathbf{U}^{*}\right)\right) \partial_{s} \Phi\left(s ; \mathbf{U}_{l}, \mathbf{U}^{*}\right) \mathrm{d} s \\
\sigma_{2}\left(\mathbf{U}_{r}-\mathbf{U}^{*}\right) & =\int_{0}^{1} A\left(\Phi\left(s ; \mathbf{U}^{*}, \mathbf{U}_{r}\right)\right) \partial_{s} \Phi\left(s ; \mathbf{U}^{*}, \mathbf{U}_{r}\right) \mathrm{d} s .
\end{aligned}
$$

If the family of paths is explicitly known, we have again a nonlinear system to solve. Nevertheless, if the family of paths is implicitly given by the vanishing-viscosity limit of a regularized problem

$$
\mathbf{U}_{t}+A(\mathbf{U}) \mathbf{U}_{x}=\epsilon\left(R(\mathbf{U}) \mathbf{U}_{x}\right)_{x}
$$

they are given by the viscous profiles as follows: recall that a travelling wave is a solution of (A.6) of the form

$$
\mathbf{U}(x, t)=\varphi\left(\frac{x-\sigma t}{\epsilon}\right)
$$

satisfying the conditions:

$$
\lim _{\xi \rightarrow-\infty} \varphi(\xi)=\mathbf{U}_{-}, \quad \lim _{\xi \rightarrow \infty} \varphi(\xi)=\mathbf{U}_{+}, \quad \lim _{\xi \rightarrow- \pm \infty} \varphi^{\prime}(\xi)=0 .
$$

The viscous profile $\varphi$ is thus the solution of the ODE system:

$$
-\sigma \varphi^{\prime}+A(\varphi) \varphi^{\prime}-\left(R(\varphi) \varphi^{\prime}\right)^{\prime}=0
$$

with the boundary conditions (A.8). By integrating (A.9) and taking into account (A.8) we obtain

$$
\sigma\left(\mathbf{U}_{+}-\mathbf{U}_{-}\right)=\int_{-\infty}^{\infty} A(\varphi(\xi)) \varphi^{\prime}(\xi) d \xi
$$

which is the jump condition consistent with the viscous regularization of the problem. In other words, a pair of states $\left(\mathbf{U}_{-}, \mathbf{U}_{+}\right)$can be linked by an admissible shock if there exists a viscous profile satisfying (A.9) and (A.8) and, in this case, the path connecting them is, after a reparameterization, the viscous profile $\varphi$.

In most cases, the ODE system (A.9) cannot be explicitly computed and thus its solution has to be numerically approximated. We consider here the centered second-order finite difference method:

$$
\begin{aligned}
& -\sigma \frac{\varphi_{j+1}-\varphi_{j-1}}{2 \Delta \xi}+A\left(\varphi_{j}\right) \frac{\varphi_{j+1}-\varphi_{j-1}}{2 \Delta \xi}-\frac{R_{j+1 / 2}\left(\varphi_{j+1}-\varphi_{j}\right)-R_{j-1 / 2}\left(\varphi_{j}-\varphi_{j-1}\right)}{\Delta \xi^{2}}=0, \quad j=1, \ldots, N-1, \\
& \varphi_{0}=\mathbf{U}_{-}, \quad \varphi_{N}=\mathbf{U}_{+},
\end{aligned}
$$

with

$$
R_{j+1 / 2}=R\left(\bar{\varphi}_{j+1 / 2}\right)
$$

The computational domain is $[-L / 2, L / 2]$ and $\Delta \xi=L / N . \varphi_{j}$ is thus the approximation of the viscous profiles at $\xi_{j}=-L / 2+j \Delta \xi$. Once the viscous profile has been approached, the integral in (A.10) is computed using 
the composite trapezodial rule

$$
\sum_{j=1}^{N-1} A\left(\varphi_{j}\right) \frac{\varphi_{j+1}-\varphi_{j-1}}{2} .
$$

To approximate the solution of the Riemann problem (A.3), we solve the $4 N \times 4 N$ system given by:

$$
\begin{aligned}
\sigma_{1}\left(\mathbf{U}^{*}-\mathbf{U}_{l}\right) & =\sum_{j=1}^{N-1} A\left(\varphi_{j}^{1}\right) \frac{\varphi_{j+1}^{1}-\varphi_{j-1}^{1}}{2}, \\
\sigma_{2}\left(\mathbf{U}_{r}-\mathbf{U}^{*}\right) & =\sum_{j=1}^{N-1} A\left(\varphi_{j}^{2}\right) \frac{\varphi_{j+1}^{2}-\varphi_{j-1}^{2}}{2}
\end{aligned}
$$

where $\varphi_{j}^{1}, j=0, \ldots, N$ satisfy (A.11) with $\sigma=\sigma_{1}, \mathbf{U}_{-}=\mathbf{U}_{l}$, and $\mathbf{U}_{+}=\mathbf{U}^{*}$, and $\varphi_{j}^{2}, j=0, \ldots, N$ satisfy (A.11) with $\sigma=\sigma_{2}, \mathbf{U}_{-}=\mathbf{U}^{*}$, and $\mathbf{U}_{+}=\mathbf{U}_{r}$.

This system is solved by an iterative algorithm. A good initial guess can be obtained by computing first an approximated solution of the Riemann problem using a standard path-conservative method based, for instance, on the family of straight segments. This solution will provide an initial guess of the shock speeds and the intermediate states. Moreover, it will also provide relevant information about the structure of the Riemann solution.

In the general case, a viscous profile per shock needs to be approximated. In addition, the Riemann invariants are used for rarefactions or contact discontinuities, either exactly of approximately. In the latter case, the nonlinear system to be solved is much larger but still feasible.

Observe that the approximation of the viscous profiles involves two errors: first the domain is truncated to a finite length and second, the differential equation is discretized by finite differences. In order to test the convergence of the procedure, it is necessary to apply it to a nonconservative system for which the exact solutions of the Riemann problems (once the family of paths has been chosen) can be computed. This is not the case for the two-layer shallow water system. Hence, we consider the nonconservative coupled Burgers system proposed in [5]:

$$
\begin{aligned}
u_{t}+u(u+v)_{x} & =0, \\
v_{t}+v(u+v)_{x} & =0 .
\end{aligned}
$$

To fix the concept of weak solution, we consider the system as the vanishing-viscosity limit of the regularized system:

$$
\begin{aligned}
u_{t}+u(u+v)_{x} & =\epsilon u_{x x}, \\
v_{t}+v(u+v)_{x} & =\epsilon v_{x x} .
\end{aligned}
$$

See [4] for a notion of weak solution based on a different regularization.

Note that adding the two equations leads to the viscous Burgers equation for $w=u+v$. This fact allows us to exactly compute the viscous profiles of this system: given a pair of states $\left(u_{l}, v_{l}\right),\left(u_{r}, v_{r}\right)$ such that $w_{l}:=u_{l}+v_{l}>w_{r}:=u_{r}+v_{r}$, first the viscous profile of the viscous Burgers equations for $\left(w_{l}, w_{r}\right)$ is computed by solving

$$
-\sigma \varphi^{\prime}+\varphi \varphi^{\prime}-\varphi^{\prime \prime}=0,
$$

with

$$
\sigma=\frac{w_{l}+w_{r}}{2} .
$$


Next, the equations

$$
-\sigma \varphi_{u}^{\prime}+\varphi_{u} \varphi^{\prime}-\varphi_{u}^{\prime \prime}=0
$$

and

$$
-\sigma \varphi_{v}^{\prime}+\varphi_{v} \varphi^{\prime}-\varphi_{v}^{\prime \prime}=0
$$

with initial conditions

$$
\varphi_{u}(-\infty)=u_{l}, \quad \varphi_{v}(-\infty)=v_{l},
$$

respectively, are solved. The pair $\left(u_{l}, v_{l}\right)$ can be linked to

$$
\left(u^{*}=\varphi_{u}(\infty), v^{*}=\varphi_{v}(\infty)\right),
$$

by an admissible shock and after a reparameterization, the path connecting them is $\left(\varphi_{u}, \varphi_{v}\right)$.

Remark A.1. If $u_{l} \neq 0$, it can be easily checked that $\varphi_{u}$ solves (A.16) with initial condition $\varphi_{u}(-\infty)=u_{l}$ if, and only if

$$
\varphi_{v}:=\frac{v_{l}}{u_{l}} \varphi_{u}
$$

solves (A.17) with initial condition $\varphi_{v}(-\infty)=v_{l}$. Therefore, the following equality holds:

$$
v^{*}=\frac{v_{l}}{u_{l}} u^{*}
$$

Moreover, the integral appearing at the right-hand side of the first generalized Rankine-Hugoniot condition is given by

$$
\int_{-\infty}^{\infty} \varphi_{v}(\xi) \varphi_{u}^{\prime}(\xi) d \xi=\frac{v_{l}}{u_{l}} \frac{\left(u^{*}\right)^{2}-u_{l}^{2}}{2}=\frac{v_{l}+v^{*}}{2}\left(u^{*}-u_{l}\right)
$$

where (A.18) has been used. Analogously, the integral appearing in the second jump condition is given by

$$
\int_{-\infty}^{\infty} \varphi_{u}(\xi) \varphi_{v}^{\prime}(\xi) d \xi=\frac{u_{l}+u^{*}}{2}\left(v^{*}-v_{l}\right)
$$

Observe that the jump conditions are identical to the ones obtained if the family of straight segments is used. Therefore, in this case the family of straight segments also leads to the correct jump conditions.

Let us consider the initial data $\mathbf{U}_{l}=(7.99,11.01), \mathbf{U}_{r}=(0.25,0.75)$. The solution of the Riemann problem (A.3) consists of a stationary contact discontinuity $\left(\sigma_{1}=0\right)$, a shock with speed $\sigma_{2}=10$, and the intermediate state $\mathbf{U}^{*}=(4.75,14.25)$. The viscous profiles for the shock are given (up to a translation) by

$$
\varphi_{u}(\xi)=\frac{4.75}{19}\left(10-9 \tanh \frac{9}{2 \xi}\right), \quad \varphi_{v}(\xi)=\frac{14.25}{19}\left(10-9 \tanh \frac{9}{2 \xi}\right)
$$




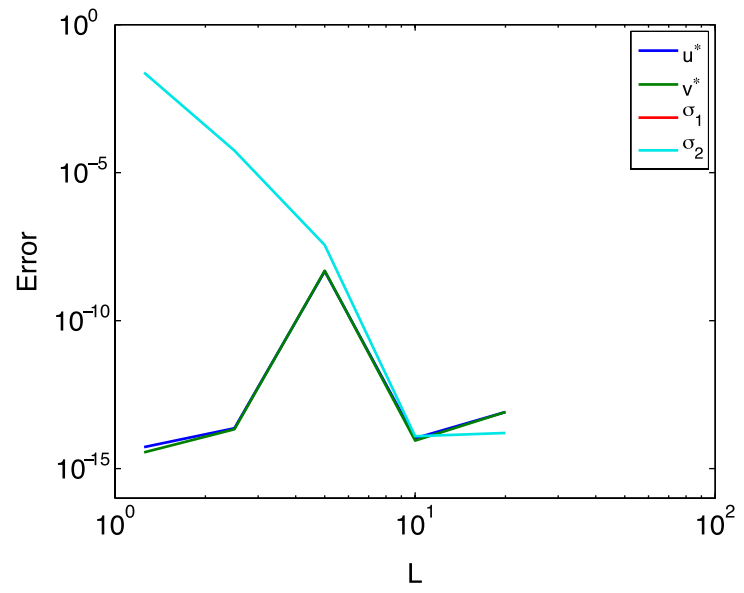

(a) Convergence study where $L \Delta \xi=0.1543$

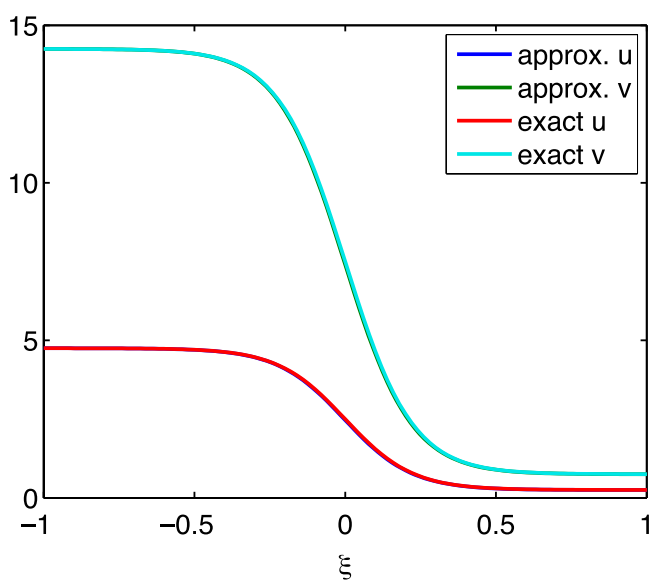

(b) $L=10, L \Delta \xi=0.1543$

Figure A.1. Approximate and exact viscous profiles for the coupled Burgers equations.

We apply now the above described ansatz to approximate this solution. In this case, instead of two shocks, there is a stationary contact discontinuity $\left(\sigma_{1}=0\right)$ and a shock. Through the contact discontinuity the Riemann invariance

$$
u_{l}+v_{l}=u^{*}+v^{*}
$$

is satisfied. Therefore, the nonlinear system to be solved is now

$$
\begin{aligned}
& u_{l}+v_{l}=u^{*}+v^{*} \\
& \sigma_{2}\left(\mathbf{U}_{r}-\mathbf{U}^{*}\right)=\sum_{j=1}^{N-1} A\left(\varphi_{j}^{2}\right) \frac{\varphi_{j+1}^{2}-\varphi_{j-1}^{2}}{2}
\end{aligned}
$$

where $\varphi_{j}^{2}, j=0, \ldots, N$ satisfy (A.11) with $\sigma=\sigma_{2}, \mathbf{U}_{-}=\mathbf{U}^{*}, \mathbf{U}_{+}=\mathbf{U}_{r}$, and $R=I d$.

In Figure A.1, a convergence study is shown. $\Delta \xi$ is decreased and $L$ increased at the same time such that both errors are reduced, while their product remains constant. Observe that both the errors in the intermediate state and the shock speed are of order of machine precision for $L=10$. The approximated viscous profile and the exact viscous profile are also plotted: they match pretty well up to a shift in $\xi$ direction. In summary, the shock relations and intermediate states are approximated quite well for a sufficiently large $L$ and a sufficiently small $\Delta \xi$, showing that this approach can be used to construct reference solutions to Riemann problems.

Although system (A.15) admits the entropy pair

$$
S=\frac{(u+v)^{2}}{2}, \quad Q=\frac{(u+v)^{3}}{6},
$$

the entropy function is not strictly convex and thus the method introduced here cannot be applied to this system.

Acknowledgements. The research of both A.H. and S.M. was funded in part by ERC STG. NN 306279 SPARCCLE. C.P. was partially supported by the Spanish Government and FEDER through the Research project MTM2015-70490-C2-1-R, and by the Andalusian Government through the project P11-FQM8179. 


\section{REFERENCES}

[1] R. Abgrall and S. Karni, A comment on the computation of nonconservative products. J. Comput. Phys. 45 (2010) $382-403$.

[2] T.J. Barth, Numerical methods for gas-dynamics systems on unstructured meshes, in An Introduction to Recent Developments in Theory and Numerics of Conservation Laws. Vol. 5 of Lecture Notes in Computational Science and Engineering, D. Kroner, M. Ohlberger and C. Rohde, eds. Springer, Berlin (1999) 195-285.

[3] A. Beljadid, P.G. LeFloch, S. Mishra and C. Parés, Schemes with well-controlled dissipation. Hyperbolic systems in nonconservative form. Commun. Comput. Phys. 21 (2017) 913-946.

[4] C. Berthon, Nonlinear scheme for approximating a non-conservative hyperbolic system. C. R. Math. Acad. Sci. Paris 335 (2002) 1069-1072.

[5] M.J. Castro, J. Macías and C. Parés, A Q-scheme for a class of systems of coupled conservation laws with source term. Application to a two-layer 1-D shallow water system. ESAIM: M2AN. 35 (2001) 107-127.

[6] M.J. Castro, J.M. Gallardo and C. Parés, High order finite volume schemes based on reconstruction of states for solving hyperbolic systems with nonconservative products. Applications to shallow-water systems. Math. Comput. 75 (2006) 11031134.

[7] M.J. Castro, P.G. LeFloch, M.L. Muñoz-Ruiz and C. Parés, Why many theories of shock waves are necessary. Convergence error in formally path-consistent schemes. J. Comput. Phys. 227 (2008) 8107-8129.

[8] M.J. Castro, C. Parés, G. Puppo and G. Russo, Central schemes for nonconservative hyperbolic systems. SIAM J. Sci. Comput. 34 (2012) 523-558.

[9] M.J. Castro, U.S. Fjordholm, S. Mishra and C. Parés, Entropy conservative and entropy stable schemes for nonconservative hyperbolic systems. SIAM J. Numer. Anal. 51 (2013) 1371-1391.

[10] M.J. Castro, T. Morales de Luna and C. Parés, Well-balanced schemes and path-conservative numerical methods, in Handbook of numerical methods for hyperbolic problems. Vol. 18 of Handb. Numer. Anal. Elsevier, North-Holland, Amsterdam (2017) $131-175$.

[11] C.M. Dafermos, in Hyperbolic Conservation Laws in Continuum Physics, Vol. 325 of Grundlehren Math. Wissenschaften Series. Springer Verlag (2000).

[12] G. Dal Maso, P.G. LeFloch and F. Murat, Definition and weak stability of nonconservative products. J. Math. Pure Appl. 74 (1995) 483-548.

[13] M. Dumbser, M.J. Castro, C. Parés and E.F. Toro, ADER schemes on unstructured meshes for nonconservative hyperbolic systems: applications to geophysical flows. Comput. Fluids 38 (2009) 1731-1748.

[14] M. Dumbser, A. Hidalgo, M.J. Castro, C. Parés and E.F. Toro, FORCE schemes on unstructured meshes II: nonconservative hyperbolic systems. Comput. Meth. Appl. Mech. Eng. 199 (2010) 625-647.

[15] U.S. Fjordholm and S. Mishra, Accurate numerical discretizations of nonconservative hyperbolic systems. ESAIM: M2AN. 46 (2012) 187-296.

[16] U.S. Fjordholm, S. Mishra and E. Tadmor, Arbitrary order accurate essentially non-oscillatory entropy stable schemes for systems of conservation laws. SIAM J. Numer. Anal. 50 (2012) 544-573.

[17] A. Hiltebrand, Entropy-stable discontinuous Galerkin finite element methods with streamline diffusion and shock-capturing for hyperbolic systems of conservation laws. Ph.D. thesis, ETH Zurich, No. 22279 (2014).

[18] A. Hiltebrand and S. Mishra, Entropy stable shock-capturing space-time discontinuous Galerkin schemes for systems of conservation laws. Numer. Math. 126 (2014) 103-151.

[19] A. Hiltebrand and S. Mishra, Efficient preconditioners for a shock-capturing space-time discontinuous Galerkin schemes for systems of conservation laws. Commun. Comput. Phys. 17 (2015) 1360-1387.

[20] T.Y. Hou and P.G. LeFloch, Why nonconservative schemes converge to wrong solutions. Error analysis. Math. Comput. 62 (1994) 497-530.

[21] J. Jaffre, C. Johnson and A. Szepessy, Convergence of the discontinuous Galerkin finite element method for hyperbolic conservation laws. Math. Model. Meth. Appl. Sci. 5 (1995) 367-386.

[22] C. Johnson and A. Szepessy, On the convergence of a finite element method for a nonlinear hyperbolic conservation law. Math. Comput. 49 (1987) 427-444.

[23] S. Karni, Viscous shock profiles and primitive formulations. SIAM J. Numer. Anal. 29 (1992) 1592-1609.

[24] P.G. LeFloch, in Hyperbolic Systems of Conservation Laws: The Theory of Classical and Nonclassical Shock Waves. Lecture Notes in Mathematics. ETH Zürich, Birkhäuser (2002).

[25] P.G. LeFloch and S. Mishra, Numerical methods with controlled dissipation for small-scale dependent shocks. Acta Numer. 23 (2014) 743-816.

[26] C. Parés, Numerical methods for nonconservative hyperbolic systems: a theoretical framework. SIAM J. Numer. Anal. 44 (2006) 300-321.

[27] S. Rhebergen, O. Bokhove and J.J.W. van der Vegt, Discontinuous Galerkin finite element methods for hyperbolic nonconservative partial differential equations. J. Comput. Phys. 227 (2008) 1887-1922.

[28] E. Tadmor, Entropy stability theory for difference approximations of nonlinear conservation laws and related time-dependent problems. Acta Numer. 12 (2003) 451-512. 